Taboada, M. de los Á., M. J. Miranda \& I. J. C. Gavriloff. 2021. Primer relevamiento de la riqueza algal de una laguna de montaña del Noroeste Argentino. Bonplandia 30(1): 37-60. http://dx.doi.org/10.30972/bon.3014589

Recibido 11 Julio 2020. Aceptado 27 Agosto 2020.

Publicado en línea: 23 de diciembre 2020. Publicado impreso: 15 de febrero 2021

ISSN 0524-0476 impreso. ISSN 1853-8460 en línea.

\title{
Primer relevamiento de la riQueza algal de una laguna de montaña del Noroeste Argentino
}

First survey of the algal richness of a Nordwestern Argentinean mountain pond

\author{
María de los Á. Taboada ${ }^{1,2}$, María J. Miranda ${ }^{3,4}$ - y Igor J. C. Gavriloff ${ }^{3}(\mathbb{C}$
}

\begin{abstract}
Resumen: El Noroeste de Argentina presenta una amplia variedad de sistemas de aguas continentales incluidas lagunas irregulares y con dimensiones variables muchas de las cuales se han formado por deslizamientos de laderas. Las lagunas de altura son escasas, de distribución heterogénea y constituyen ecosistemas frágiles, vulnerables a la intervención humana y a efectos naturales. Estos ecosistemas leníticos, son inestables y de vida corta, por lo que su estudio es de relevancia para diversos fines. Debido a la pérdida acelerada de hábitats y extinciones de la biota, en los últimos años hubo un importante interés en revalorizar y estudiar la biodiversidad. En este contexto mundial y dado que existen escasos trabajos en la zona de estudio, se realizó un análisis de la riqueza y composición del fitoplancton en la laguna del Siambón, ubicada en las Yungas del Noroeste Argentino. Se determinaron 39 especies: 2 algas verdes, 5 algas verdes-azules (cianobacterias) y 32 diatomeas. Se presentan dos nuevas citas de diatomeas para la provincia de Tucumán (Nitzschia inconspicua y Stauroneis anceps). Esta primera contribución ficológica en este sistema servirá como línea de base para futuros estudios limnológicos, ficológicos y paleoambientales. Asimismo, constituye un aporte para incrementar el conocimiento de la diversidad biológica en lagunas de altura.
\end{abstract}

Palabras clave: Fitoplancton, laguna, NOA, Yungas.

Summary: Northwest Argentina has a wide variety of inland water systems including irregular ponds of variable dimensions, many of which have been formed by landslides. High-altitude ponds are scarce, of heterogeneous distribution and constitute fragile ecosystems, vulnerable to human intervention and natural effects. These lenitic ecosystems are unstable and shortlived, so their study is relevant for various purposes. Due to the accelerated loss of habitats and extinctions of biota, in recent years there has been an important interest in revaluing and studying biodiversity. In this global context and given the scarcity of research in the study area, an analysis of the richness and composition of phytoplankton was carried out in the Siambón pond, located in the Yungas of Northwest Argentina. 39 species were determined: 2 green algae, 5 green-blue algae (cyanobacteria) and 32 diatoms. Two new diatom citations are presented for Tucumán province (Nitzschia inconspicua and Stauroneis anceps). This first phycological contribution to this system will serve as a baseline for future limnological, phycological and palaeoenvironmental studies. Likewise, it constitutes a contribution to increase the knowledge of biological diversity in high altitude ponds.

Key words: NOA, phytoplankton, ponds, Yungas.

\footnotetext{
${ }^{1}$ Instituto de Ecosistemas de Aguas Continentales, Fundación Miguel Lillo-Miguel Lillo 251, T4000JFE, San Miguel de Tucumán, Argentina. E-mail: mtaboada@lillo.org.ar

${ }^{2}$ Unidad Ejecutora Lillo UEL-CONICET.

${ }^{3}$ Facultad de Ciencias Naturales e IML-UNT. Miguel Lillo 205 T4000JFE-San Miguel de Tucumán, Argentina

${ }^{4}$ Instituto de Biodiversidad Neotropical, IBN (CONICET-UNT)- T4107JFE -Yerba Buena, Tucumán, Argentina
} 


\section{Introducción}

En la Argentina es posible encontrar una amplia variedad de lagos y lagunas en relación a su formación, tamaño, forma, química y fertilidad natural. La región del Noroeste Argentino (NOA en adelante) presenta numerosos y diversos sistemas de aguas continentales (ríos, arroyos, lagunas y embalses). Las lagunas son cuerpos de agua generalmente pequeños, de escasa profundidad y de forma cóncava (Mancini et al., 2012), que permiten el establecimiento de organismos acuáticos o terrestres, migratorios o residentes, que logran adaptarse e incrementan su valor paisajístico (García Rodríguez et al., 2014). Sin embargo, la diversidad del agua superficial en una región depende de tres factores principales: clima, geología y relieve (Quirós et Drago, 1999). En la provincia de Tucumán, dado su relieve montañoso, los movimientos en masa constituyen uno de los procesos geomorfológicos más importantes. La percepción de estos eventos se ha incrementado en los últimos años como consecuencia de la generación de grandes flujos acontecidos en respuesta a fenómenos hidrológicos de gran magnitud que dan lugar a la formación de lagunas o endicamientos naturales (Fernández, 2009). Las lagunas formadas por deslizamiento de terreno son irregulares, puede alcanzar grandes dimensiones, son inestables y de vida corta (Wetzel, 1981). Las lagunas de altura originadas por deslizamientos son enclaves biológicos de gran valor por su alta biodiversidad y rareza, además por su situación biogeográfica se convierten en rutas migratorias de numerosas especies de aves y sobre todo cumplen un papel relevante como sistemas de inestimable complejidad y valor ecológico (Casado et Montes, 1995). Desde el punto de vista paleoambiental, las lagunas son reservorios de información de gran utilidad para indagar acerca de cambios hidrológicos y climáticos a partir de análisis multi-proxies o de múltiples indicadores ambientales. La laguna, objetivo de este estudio, se halla ubicada en la localidad de El Siambón (Tucumán) y pertenece a la cuenca del río Potrerillos, dicho sistema presenta evidencias actuales de procesos de remoción en masa que se encuentran afectando las características hidrológicas de la misma a partir de la formación de diques naturales como consecuencia del ingreso de grandes masas de sedimentos desplazadas de manera súbita dentro de los cauces (Fernández, 2009).

En los últimos años hubo un renovado interés en valorizar y estudiar la biodiversidad, dado que la misma se halla amenazada por el cambio climático, las variaciones en el uso del suelo, la incorporación de las especies invasoras, la sobreexplotación, la contaminación y los cambios en la población humana (Sala et al., 2000). A nivel global, los ecosistemas subtropicales de montaña junto con la biodiversidad que albergan, se encuentran entre las regiones más vulnerables a estos efectos (Raxworthy et al., 2008; Chen et al., 2009; Foden et al., 2013). La pérdida de biodiversidad no solo vuelve a los ecosistemas más susceptibles, sino que altera los procesos y cambia la resiliencia de los ecosistemas al cambio ambiental (Chapin et al., 2000). Es así que resulta relevante el descubrimiento del mayor número de especies no conocidas antes de que desaparezcan y no se tenga oportunidad de registrarlas. El entendimiento de esta biodiversidad a través de estudios morfológicos, filogenéticos, taxonómicos y el conocimiento integrado y multidisciplinario, es necesario para permitir un manejo más adecuado de la riqueza del planeta. Sin embargo, esta preocupación parece estar principalmente orientada hacia los bosques tropicales, desiertos y otros ecosistemas terrestres y poca importancia se ha puesto en los ecosistemas acuáticos (John, 1994). Solamente en el caso de las algas, como organismos típicamente acuáticos en la actualidad las descritas alcanzarían la cifra de 40.000 (Groombridge, 1992), de las cuales entre 6.500 y 7.000 corresponden a especies marinas (Silva, 1992). La estimación de la diversidad algal ha sido un punto de debate que aún continua y que difícilmente será resuelto (John, 1994; Pedroche, 2000; Silva, 1992).

El fitoplancton es la taxocenosis de organismos autótrofos que viven en suspensión en la columna de agua (Reynolds, 2006), de gran importancia en el funcionamiento de los ecosistemas acuáticos siendo responsable de la mitad de la producción primaria del planeta (Falkowski et al., 2003; Field et al., 1998). El número de especies presentes en un cuerpo de agua y la abundancia de cada una de ellas brinda un indicio del grado de complejidad del ambiente. Los cuerpos de agua con muchas especies de algas generalmente soportan una 
fauna también abundante $\mathrm{y}$, por lo tanto, las relaciones ecológicas, incluyendo el pastoreo, en ellos tienden a ser estables y complejas. En este contexto mundial de ampliar los conocimientos de la biodiversidad, particularmente de las algas y debido a que no existen estudios previos, el objetivo de este trabajo es aportar al conocimiento de la riqueza específica del fitoplancton en una laguna de Yungas del Noroeste Argentino.

\section{Materiales y Métodos}

\section{Área de Estudio}

La zona de estudio se encuentra dentro de las estribaciones orientales de las Cumbres Calchaquíes y tiene como límites norte y sur a las cuencas de los ríos Tipas y Lules respectivamente y como límite oeste y este las Sierras del Siambón y las Cumbres del Periquillo, respectivamente. Hacia el este, se ubica el valle de San Javier y a continuación el piedemonte ondulado que culmina en los límites del Gran San Miguel de Tucumán.

La laguna a la que designaremos toponímicamente como Laguna del Siambón, pertenece a la cuenca del río Potrerillos, encontrándose ubicada en un afluente del mismo denominado río del Agua Salada (2642’38,64'S y 65²4'58,00”'W, 1266 m s.n.m.) (Fig. 1). El sistema presenta evidencias geológicas, tanto actuales como Cuaternarias, de procesos de remoción en masa que se encuentran afectando las características hidrológicas de la misma a partir de la formación de diques naturales como consecuencia del ingreso de grandes masas de sedimentos desplazadas de manera súbita dentro de los cauces (Moyano, 2006; Fernández, 2009).

Desde el punto de vista ambiental, el área de estudio se encuentra localizada dentro de la Ecorregión de Yungas (Olson, 2001). Estas selvas constituyen el extremo austral de una franja de selvas y bosques de montaña que se extienden en las laderas orientales húmedas de los Andes desde Colombia hasta el Noroeste de Argentina (Lomáscolo et al., 2014). Las selvas de montaña -o Yungas- se caracterizan por ser una unidad de gran importancia regional, por la elevada diversidad biológica que poseen, y por su papel en la regulación hídrica y protección de cuencas. Entre los factores que las caracterizan se destacan las lluvias de tipo orográfico, que se dan gracias a la altura de los cordones montañosos, los cuales ejercen un efecto importante sobre la distribución espacial e intensidad de las precipitaciones, condicionando la distribución
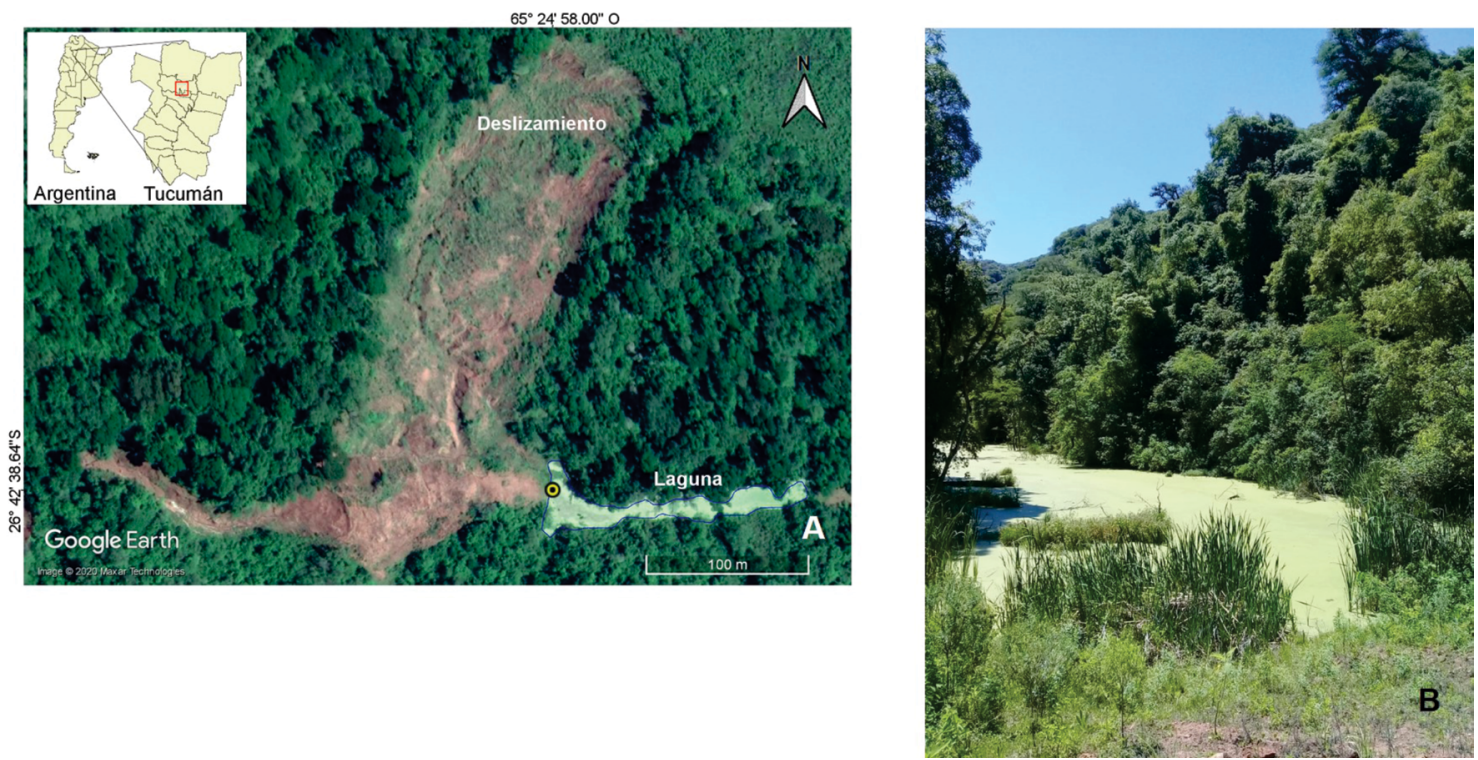

Fig. 1. Área de estudio. A: Imagen satelital del deslizamiento y de la laguna. B: Fotografía de la laguna.

Fig. 1. Study area. A: Satellite image of the landslide and the ponds. B: Photograph of the pond. 
y exuberancia de su vegetación. En general, las Yungas se desarrollan a lo largo de un gradiente altitudinal que va de los 400 a los 3.000 m s.n.m. (Quiroga et Grau, 2020), lo que les otorga una gran diversidad de microclimas en base a la variación de precipitaciones (1000-3000 mm anuales) y la temperatura a diferentes alturas (Grau, 2010). En particular, los valles de Raco y El Siambón se encuentran fundamentalmente en el piso altitudinal de Selva Nublada, pero poseen sectores con vegetación característica del piso más alto de Yungas, los pastizales de Neblina (Viollaz et al., 2015). El clima subtropical monzónico caracteriza a esta zona, determinando la ocurrencia del $80 \%$ de la precipitación anual entre los meses de noviembre a marzo (veranos húmedos e inviernos secos).

\section{Trabajo de campo y laboratorio}

Se realizó un muestreo ficológico en la primavera de 2019, mediante una red de plancton de $20 \mu \mathrm{m}$ de tamaño de poro para la obtención de cuatro muestras cualitativas, que fueron conservadas en frascos plásticos y fijadas in situ con Lugol para una mejor preservación. Para los análisis cualitativos de las diatomeas se procedió a la eliminación de la materia orgánica según la metodología propuesta por Battarbee (1986) mediante peróxido de hidrógeno y calor. Para realizar los preparados permanentes se utilizó resina Naphrax ${ }^{\circledR}$ como medio de montaje y fueron observados con microscopio binocular Zeiss Lab Axio 1 que dispone de cámara fotográfica y un software que permite realizar mediciones. Las características morfométricas consideradas para las algas verdeazuladas fueron: largo (L) y ancho (1) de: filamentos, células vegetativas, heterocistos y/o acinetos, células apicales y dimensiones de las colonias. Para las especies de algas verdes se midió el diámetro de las colonias y de las células. En las diatomeas se tuvieron en cuenta: longitud del eje apical y transapical, presencia y forma del rafe, el número de estrías, fíbulas y/o costillas en 10 micras, la presencia de estigmas, si tenían poros, aréolas o alguna otra característica particular.

Se realizaron recuentos fitoplanctónicos por frecuencias relativas según la técnica propuesta por Villafañe et Freid (1995). Siguiendo a Luque (1998) se reemplazó la cámara de SedgwickRafter por un cubreobjetos de $24 \times 50 \mathrm{~mm}$. Se prepararon cinco cámaras de cada muestra por para lo cual se tomaron una alícuota de $0,2 \mathrm{ml}$ de esta. Los recuentos se llevaron a cabo mediante 3 transectas horizontales equidistantes evitando los efectos de borde y las cámaras fueron observadas en 40x a 500 aumentos.

Para las determinaciones taxonómicas se consultó: para Cianofitas a Frémy (1930); Desikachary (1959); Starmach (1966), Anagnostidis et Komárek (1988) y Komárek et Anagnostidis $(1999 ; 2005)$. En el caso de las Chlorophyta se siguió a Prescott (1961) y Tracanna (1982, 1985). Para las diatomeas se consultó a: Patrick (1961), Patrick et Reimer (1966, 1975), Hartley (1996), Krammer et Lange-Bertalot (1986, 1988, 1991, 2000, 2004), Metzeltin et Lange-Bertalot (1998, 2007), Lange-Bertalot (1999), Rumrich et al. (2000), Metzeltin et al. (2005), la base de datos algaebase (Guiry et Guiry, 2020) y publicaciones diatomológicas de autores varios. Para la distribución geográfica en Argentina se consultó a Luchini et Verona (1972), Vouilloud (2003) y publicaciones específicas de autores varios.

Las muestras fueron recolectadas en la provincia de Tucumán, Dep. Tafí Viejo, en Laguna del Siambón (26²2’38,64”'S y 65²4'58,00”W) el 16/11/2019 e incorporadas a la Colección Ficológica (LIL) de la Fundación Miguel Lillo, bajo numeración de la primer autora: Taboada M.A.: 25.345, 25.346, 25.347 y 25.348 .

\section{Resultados}

Se determinaron un total de 39 especies pertenecientes a las Divisiones Chlorophyta o algas verdes (2), Cyanophyta o algas verdeazuladas (5) y Heterokontophyta-Clase Bacillariophyceae o diatomeas (32). Dentro de las diatomeas se presentan dos nuevas citas para la provincia de Tucumán: Nitzschia inconspicua y Stauroneis anceps (indicadas con * en el texto y en la Tabla 1). El listado taxonómico y el porcentaje de las frecuencias relativas se presentan en la Tabla 1.

A continuación, se detallan los registros de especies con sus descripciones, valores morfométricos, distribución y algunas características ecológicas. 
Tabla 1. Listado taxonómico de las microalgas observadas en la Laguna del Siambón y sus porcentajes de frecuencias relativas. "Primeros registros para la provincia de Tucumán.

Table 1. Taxonomic listing of the microalgae observed in the Siambón pond and their percentages of relative frequencies. * First records for the province of Tucumán.

\begin{tabular}{|c|c|c|c|c|}
\hline Especies & $\%$ o & Frecuer & cia relati & \\
\hline Div. Cyanophyta & $100-60 \%$ & $60-45 \%$ & $45-20 \%$ & $>20 \%$ \\
\hline Homoeothrix juliana (Bornet \& Flahault ex Gomont) Kirchner & & $\mathrm{x}$ & & \\
\hline Merismopedia glauca (Ehrenberg) Kützing & & & $\mathrm{x}$ & \\
\hline Nostoc commune Vaucher ex Bornet \& Flahault & & $\mathrm{x}$ & & \\
\hline Oscillatoria limosa C. Agardh ex Gomont & & & $x$ & \\
\hline $\begin{array}{l}\text { Phormidium chalybeum (Mertens ex Gomont) Anagnostidis \& } \\
\text { Komarek }\end{array}$ & & & & $\mathrm{x}$ \\
\hline Div. Chlorophyta & & & & \\
\hline Coelastrum microporum Nägeli & & $x$ & & \\
\hline Pandorina morum (O. F. Müller) Bory & & $x$ & & \\
\hline Div. Heterokontophyta - Clase Bacillariophyceae & & $x$ & & \\
\hline Achnanthes coarctata (Brébisson ex W. Smith) Grunow & & $\mathrm{x}$ & & \\
\hline Amphipleura lindheimeri Grunow & $\mathrm{x}$ & & & \\
\hline Amphora copulata (Kützing) Schoeman \& R. E. M. Archibald & & & $x$ & \\
\hline Caloneis bacillum (Grunow) Cleve & & & $x$ & \\
\hline Cocconeis placentula var. euglypta (Ehrenberg) Grunow & $\mathrm{x}$ & & & \\
\hline Cocconeis placentula var. lineata (Ehrenberg) Van Heurck & $\mathrm{x}$ & & & \\
\hline Denticula elegans Kützing & & $\mathrm{x}$ & & \\
\hline Denticula kuetzingii Grunow & & & $x$ & \\
\hline Diploneis puella (Schumann) Cleve & & & & $x$ \\
\hline Epithemia sorex Kützing & & $\mathrm{x}$ & & \\
\hline Fallacia pygmaea (Kützing) Stickle \& D. G. Mann & $\mathrm{x}$ & & & \\
\hline Gomphonema affine Kützing & & $x$ & & \\
\hline Gomphonema affinopsis Metzeltin \& García-Rodríguez & & & $x$ & \\
\hline Gomphonema capitatum Ehrenberg & $\mathrm{x}$ & & & \\
\hline Gomphonema lippertii Reichardt \& Lange-Bertalot & & & & $\mathrm{x}$ \\
\hline Gomphonema parvulum (Kützing) Kützing & $x$ & & & \\
\hline Luticola kotschyi (Grunow) Taylor, Harding \& Archibald & & & $\mathrm{x}$ & \\
\hline Navicula cryptocephala Kützing & & & $\mathrm{x}$ & \\
\hline Navicula peregrina (Ehrenberg) Kützing & & $\mathrm{x}$ & & \\
\hline Navicula veneta Kützing & & & & $\mathrm{x}$ \\
\hline Nitzschia amphibia Grunow & $\mathrm{x}$ & & & \\
\hline Nitzschia dissipata (Kützing) Grunow & & & $\mathrm{x}$ & \\
\hline *Nitzschia inconspicua Grunow & & & & $x$ \\
\hline
\end{tabular}




\begin{tabular}{|c|c|c|c|c|}
\hline \multirow[b]{2}{*}{ Div. Cyanophyta } & \multicolumn{4}{|c|}{$\%$ de Frecuencia relativa } \\
\hline & $100-60 \%$ & $60-45 \%$ & $45-20 \%$ & $>20 \%$ \\
\hline Nitzschia linearis Smith & $\mathrm{x}$ & & & \\
\hline Nitzschia sigma (Kützing) Smith & & $x$ & & \\
\hline Planothidium frequentissimum (Lange-Bertalot) Lange-Bertalot & $x$ & & & \\
\hline Rhopalodia gibba (Ehrenberg) O. Müller & & $\mathrm{x}$ & & \\
\hline *Stauroneis anceps Ehrenberg & & & $x$ & \\
\hline $\begin{array}{l}\text { Stauroneis phoenicenteron (Hustedt) Krammer \& Lange- } \\
\text { Bertalot }\end{array}$ & & $\mathrm{x}$ & & \\
\hline Surirella ovalis Brèbisson & $\mathrm{x}$ & & & \\
\hline Tryblionella hungarica (Grunow) Frenguelli & & $\mathrm{x}$ & & \\
\hline Ulnaria ulna (Nitzsch) Compère & $\mathrm{x}$ & & & \\
\hline
\end{tabular}

\section{Div. Cyanophyta}

Homoeothrix juliana (Bornet \& Flahault ex Gomont) Kirchner (Fig. 2A)

Filamentos más o menos rectos o levemente curvados, solitarios o en pequeños grupos. De coloración verde azulada. Vaina muy fina, hialina y delicada. Dimensiones celulares: filamento: L: 7,5-9,5 $\mu \mathrm{m}$; 1: 5-7 $\mu \mathrm{m}$.

Ecología: En aguas dulces y termales, sobre rocas, generalmente sobre sustratos calcáreos, raramente entre otras algas o epifítico. Cosmopolita (Frémy, 1930; Komárek et Anagnostidis, 2005).

Distribución geográfica: Córdoba y Santa Cruz (Tell, 1985), Tucumán (Mirande et Tracanna, 2003; Mirande, 2006; Taboada et al., 2015).

Material estudiado: Taboada $25.345 \mathrm{y}$ 25.347 (LIL).

Merismopedia glauca (Ehrenberg) Kützing (Fig. 2D)

Colonias de medianas a grandes, usualmente rectangulares, las células se disponen regularmente en filas paralelas. Células de forma subesféricas o hemisféricas algo cuadrangulares de color verde-azuladas. Mucilago incoloro. Dimensiones celulares: L: 3,8-5 $\mu \mathrm{m} ; 1: 4,8-5,5 \mu \mathrm{m}$.

Ecología: Agua dulce, metafítica y epilítica en estanques, lagunas, pantanos, ríos, ocasionalmente ticoplanctónica, generalmente en aguas ligeramente ácidas, probablemente cosmopolita (Komárek et Anagnostidis, 1999).

Distribución geográfica: Buenos Aires y Córdoba. En el NOA en Catamarca, Salta (Tracanna, 1985) y Tucumán (Tracanna, 1985; Martínez De Marco, 2009; Taboada, 2017).

Material estudiado: Taboada 25.345 y 25.346 (LIL).

Nostoc commune Vaucher ex Bornet \& Flahault (Fig. 2B)

Los filamentos son flexuosos. La vaina es hialina. Los heterocistos presentan una coloración más clara que las células vegetativas. Los acinetos fueron escasos y de dimensiones similares a las células vegetativas. Dimensiones celulares: 1: 4-5,5 $\mu \mathrm{m}$. Heterocistos: L: 4,5-6,5 $\mu \mathrm{m}, 1: 4-5 \mu \mathrm{m}$, acinetos pocos frecuentes.

Ecologia: Agua dulce, salobre, epífitas, edáficas (Fremy, 1930)

Distribución geográfica en Argentina: Buenos Aires, Córdoba, Santa Cruz y Tierra del Fuego (Tell, 1985). En el NOA Jujuy (Guarrera et Kuhnemann, 1949) y Tucumán (Martínez De Marco, 2009; Taboada et al., 2016; 2017)

Material estudiado: Taboada 25.345 y 25.348 (LIL).

Oscillatoria limosa C. Agardh ex Gomont (Fig. 2C)

Filamentos rectos y largos o muy poco curvados, con células notablemente más 

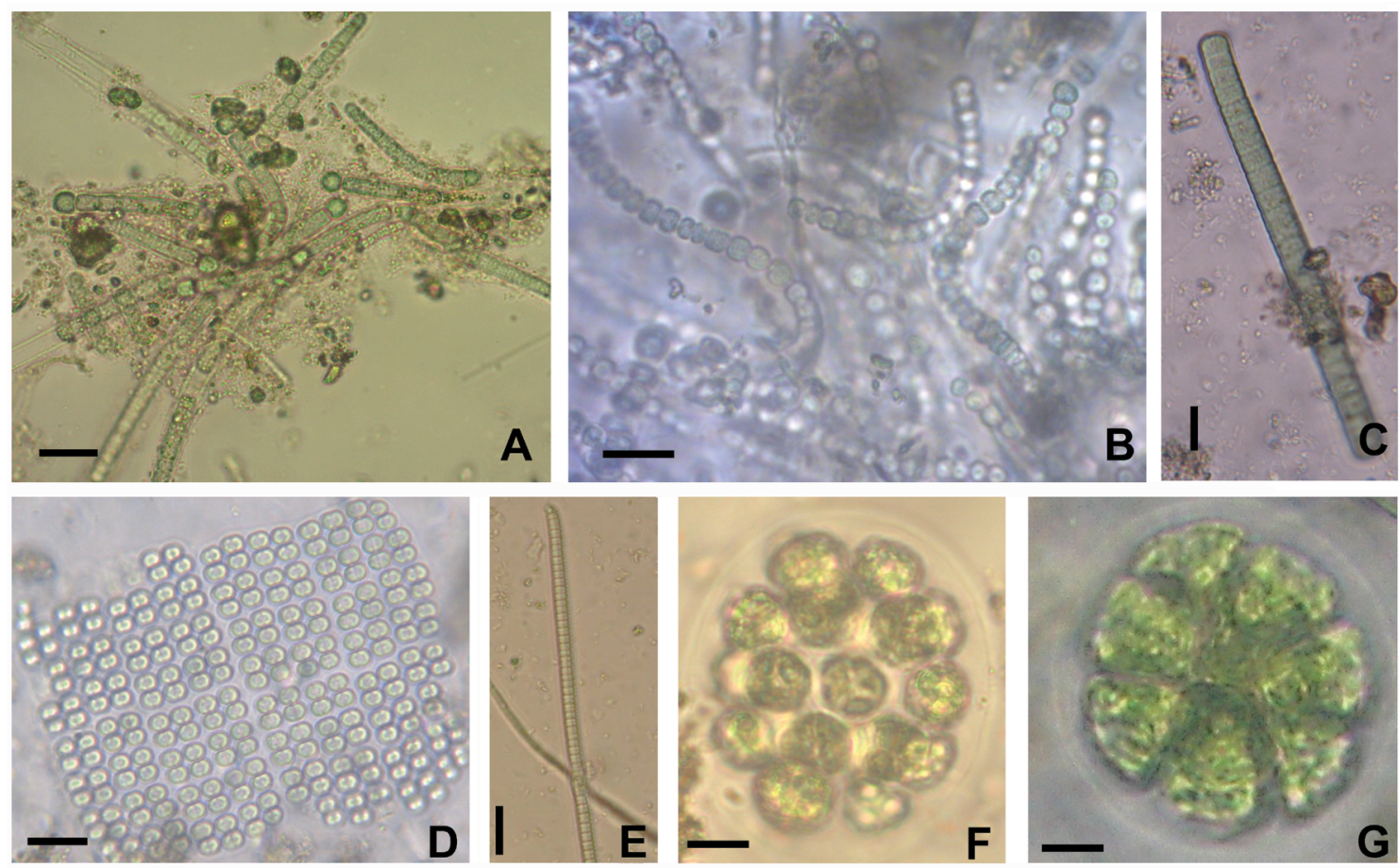

Fig. 2. Fotografías de las microalgas observadas en la laguna. Div. Cyanophyta: A: Homeotrix juliana. B: Nostoc commune. C: Oscillatoria limosa. D: Merismopedia glauca. E: Phormidium chalybeum. Div. Chlorophyta: F: Coelastrum microporum. G: Pandorina morum. La escala corresponde a $10 \mu \mathrm{m}$.

Fig. 2. Photographs of the microalgae observed in the ponds. Div. Cyanophyta: A: Homeotrix juliana. B: Nostoc commune. C: Oscillatoria limosa. D: Merismopedia glauca. E: Phormidium chalybeum. Div. Chlorophyta: F: Coelastrum microporum. G: Pandorina morum. The scale corresponds to $10 \mu \mathrm{m}$.

anchas que largas. Color verde oliváceo. Posee movimientos oscilantes suaves. Células no constrictas y finamente granulada. Las células apicales no capitadas y redondeadas. Dimensiones celulares: célula vegetativa: L: 2,5$3 \mu \mathrm{m}, 1:$ 14-14,8 $\mu \mathrm{m}$; célula apical: L: 5- 6,7 $\mu \mathrm{m}$, 1: $14 \mu \mathrm{m}$.

Ecología: Agua dulce, salobre, epífitas.

Distribución geográfica: Corrientes, Chubut, Córdoba, Buenos Aires, Río Negro, Santa Cruz y Tierra del Fuego (Tell, 1985). En el NOA: Jujuy (Tracanna, 1985) y Tucumán (Mirande, 2006). Material estudiado: Taboada 25.345 y 25.346 (LIL).

\section{Phormidium chalybeum (Mertens ex Gomont)} Anagnostidis \& Komarek (Fig. 2E)

Filamentos solitarios. No se observa vaina. De color verde amarillento claro. Célula apical roma a redondeada. Células isodiamétricas no constrictas. Dimensiones celulares: L: 3,5-4,3 $\mu \mathrm{m}$ y $1:$ : 7,5-8 $\mu \mathrm{m}$.

Ecología: Agua dulce, perifítica y ventónica. Cosmopolita (Komarek et Anagnostidis, 2005). Distribución geográfica: Catamarca (Mirande et Tracanna, 2015), Córdoba y Corrientes (Tell, 1985). En el NOA: Jujuy (Mirande et Tracanna, 1995), Salta (Salusso, 2005) y Tucumán (Mirande et Tracanna, 1995; Seeligmann, 1998; Mirande et Tracanna, 2003; Taboada, 2017).

Material estudiado: Taboada 25.345 (LIL).

\section{Div. Chlorophyta, Clase Chlorophyceae}

\section{Coelastrum microporum Nägeli (Fig. 2F)}

Cenobios esféricos o poliédricos. El número de células varía de 8 a 32 . Células individuales globosas, sin procesos interconectantes se unen 
entre sí mediante mucílago. Dimensiones: colonia de $20 \mu \mathrm{m}$ de diámetro; células de 6,89,5 $\mu \mathrm{m}$ de diámetro.

Ecología: Cosmopolita. Común en el ticoplancton de muchos lagos y charcos (Prescott, 1961).

Distribución geográfica: Antártida, Buenos Aires, Córdoba, Corrientes, Río Negro, Santa Cruz y Tierra del Fuego (Tell, 1985). En el NOA: Catamarca (Tracanna, 1985), Jujuy y Tucumán (Tracanna, 1982; Seeligmann et Tracanna, 1994; Mirande et Tracanna, 2003).

Material estudiado: Taboada 25.345 y 25.346 (LIL).

Pandorina morum (O. F. Müller) Bory (Fig. 2G)

Colonias esféricas a semiesféricas de 8-16 células, incluidas en una vaina mucilaginosa. Células biflageladas, piriformes a redondeadas. Estigma en el extremo distal de la célula. Dimensiones: colonia de 43-55 $\mu \mathrm{m}$ de diámetro. Células: L.: 13-16 $\mu \mathrm{m}$; 1: 10-14,5 $\mu \mathrm{m}$.

Ecologia: Ampliamente distribuida, en aguas desde oligotrófica a eutrófica (HuberPestalozzi, 1983).

Distribución geográfica: Buenos Aires, Chubut, Córdoba, Entre Ríos, Neuquén, Río Negro, Santa Cruz y Tierra del Fuego. En el NOA: Catamarca, Jujuy (Tracanna, 1985), Salta (Tracanna, 1985; Salusso, 1998) y Tucumán (Tracanna, 1982; 1985; Seeligmann et Tracanna, 1994; Mirande et Tracanna, 1995; Seeligmann, 1998; Seeligmann et al., 2001).

Material estudiado: Taboada 25.345 y 25.346 (LIL).

\section{Div. Heterokontophyta, Clase Bacillariophyceae}

Achnanthes coarctata (Brébisson ex W. Smith) Grunow (Fig. 3A-B)

Frústulos heterovalvares. Valva linealelíptica, constricta en la zona media, con extremos anchamente rostrados y ápices truncado-redondeados. Valva sin rafe: área axial angosta, submarginal, estrías notablemente areoladas, paralelas y rectas en la zona media de la valva, curvadas hacia los ápices. Dimensiones celulares: eje apical: 51$71 \mu \mathrm{m}$, eje transapical: 13-15 $\mu \mathrm{m}$. Estrías en la valva con rafe: $10-11$ en $10 \mu \mathrm{m}$, estrías en la valva sin rafe: 9-10 en $10 \mu \mathrm{m}$. Aréolas: 10-12 en $10 \mu \mathrm{m}$.

Ecologia: Oligohalobia indiferente, $\mathrm{pH}$ indiferente, aerófila, sobre rocas, suelos, musgos, etc. Cosmopolita de agua dulce (Patrick et Reimer, 1966; Luchini et Verona, 1972).

Distribución geográfica: Antártida, Buenos Aires, Chubut, Córdoba, La Pampa, Neuquén, San Luis, Río Negro, Santa Cruz, y Tierra del Fuego (Luchini et Verona, 1972; Vouilloud, 2003). En el NOA: Catamarca (Seeligmann et Maidana, 2003), Jujuy (Maidana et al., 1998) y Tucumán (Martínez De Marco, 2009; Taboada et al., 2015; Isasmendi, 2016; Taboada et al., 2016).

Material estudiado: Taboada 25.345 y 25.346 (LIL).

Amphipleura lindheimeri Grunow (Fig. 3C)

Valvas fusiformescon extremos redondeados. Nódulo central expandido $3 / 5$ de la longitud de valvar, con el centro levemente ensanchado. Estrías transversales y longitudinales muy delicadas y numerosas. Dimensiones celulares: eje apical: $135-164 \mu \mathrm{m}$, eje transapical: $18-25$ $\mu \mathrm{m}$. Estrías transversales: $23-25$ en $10 \mu \mathrm{m}$. Estrías longitudinales: 27-29 en $10 \mu \mathrm{m}$.

Ecología: Aguas dulces estancadas o de escasa corriente, estuarina. Cosmopolita (Luchini et Verona, 1972).

Distribución geográfica: Buenos Aires, Chaco, Córdoba, Corrientes, Entre Ríos y Santa Fe (Vouilloud, 2003). En el NOA: Tucumán (Mirande et Tracanna, 1995; Tracanna et Martínez De Marco, 1997; Mirande et Tracanna, 2003; Martínez De Marco, 2009; Taboada et al., 2015, Taboada, 2017).

Material estudiado: Taboada 25.345 y 25.346 (LIL).

Amphora copulata (Kützing) Schoeman \& R. E. M. Archibald (Fig. 3D)

Valvas con el margen ventral ligeramente cóncavo o recto, a veces con una dilatación central; extremos redondeados. Área axial angosta, curvada; área central distinguible 

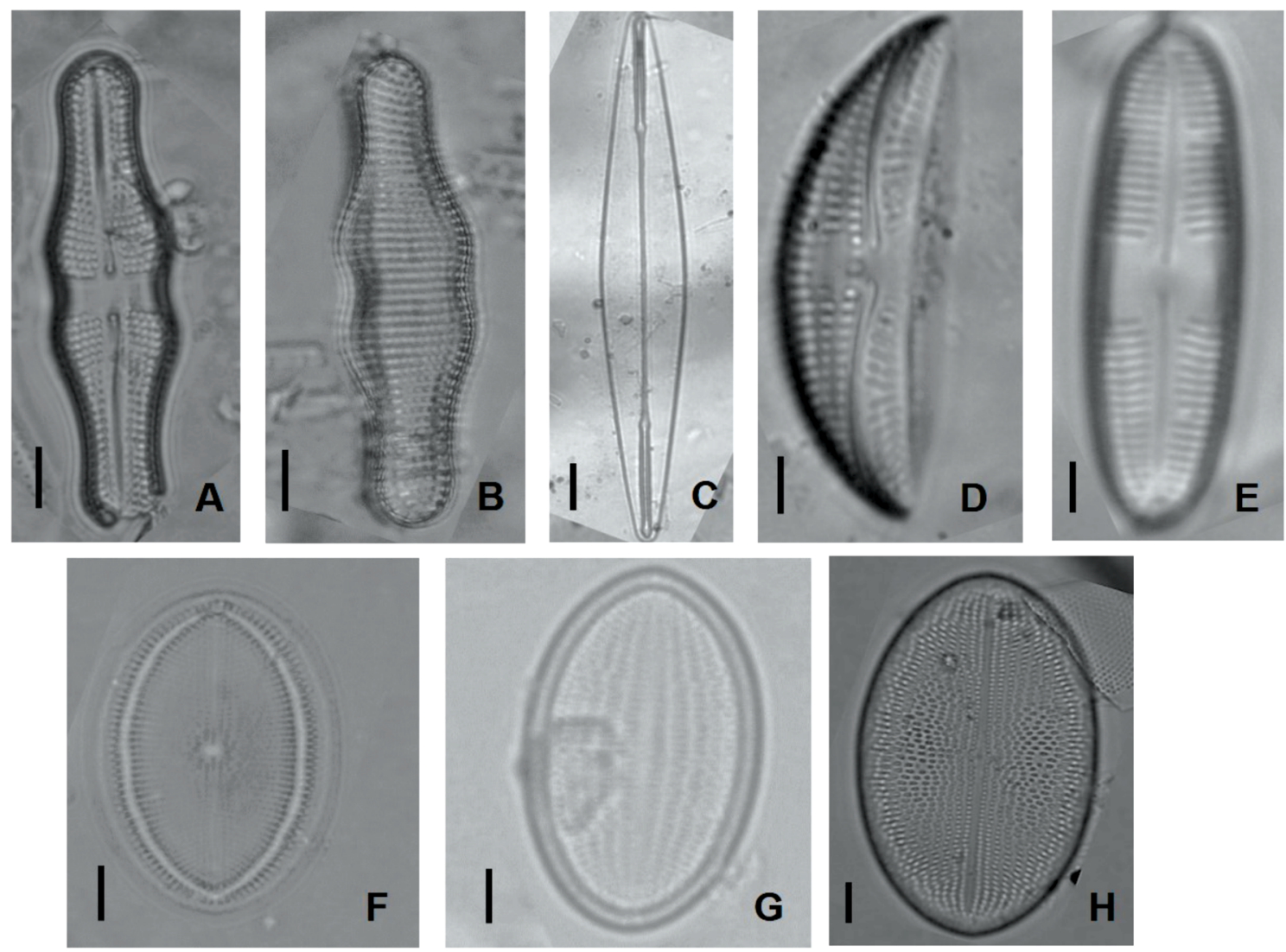

Fig. 3. Microfotografías de las diatomeas registradas en la laguna. Achnanthes coarctata. A: Valva con rafe. B: Valva sin rafe. C: Amphipleura lindheimeri. D: Amphora copulata. E: Caloneis bacillum. Cocconeis placentula var. euglypta. F: Valva con rafe. G: Valva sin rafe. H: Cocconeis placentula var. lineata. La escala corresponde a $10 \mu \mathrm{m}$.

Fig. 3. Photomicrographs of diatoms recorded in the ponds. Achnanthes coarctata. A: Valva with rafe. B: Valva without rafe. C: Amphipleura lindheimeri. D: Amphora copulata. E: Caloneis bacillum. Cocconeis placentula var. englypta. F: Valve with raphe. G: Valve without raphe. H: Cocconeis placentula var. lineata. The scale corresponds to $10 \mu \mathrm{m}$.

sólo sobre el lado ventral y con un espacio hialino cuadrangular que interrumpe las estrías dorsales en el centro de la valva. Rafe curvado, con los extremos distales y proximales dirigidos hacia el lado dorsal. Dimensiones celulares: eje apical: 20-42 $\mu \mathrm{m}$, eje transapical: 7-11 $\mu \mathrm{m}$. Estrías: $11-14$ en $10 \mu \mathrm{m}$.

Ecología: Mesotrófica a eutrófica. Especie de agua dulce, ampliamente distribuida (Levkov, 2009).

Distribución geográfica: Buenos Aires, Chaco, Córdoba, Mendoza, Misiones, Neuquén, Río Negro, San Luis, Santa Cruz y Tierra del Fuego (Vouilloud, 2003). En el NOA: Catamarca (Seeligmann et Maidana, 2003),
Jujuy (Maidana et al., 1998; Seeligmann et al., 2008), Tucumán (Seeligmann, 1998; Isasmendi et al., 2002; Taboada et al., 2015; Taboada, 2017).

Material estudiado: Taboada 25.346 y 25.347 (LIL).

Caloneis bacillum (Grunow) Cleve (Fig. 3E)

Valvas lineal-lanceoladas a lineal-elípticas con extremos cuneado-redondeados. Área axial angosta; área central formando una fascia rectangular, ancha. Rafe lateral. Estrías casi paralelas en la zona media de la valva; ligeramente radiales o convergentes en los ápices. 
Dimensiones celulares: eje apical: 9-16 $\mu \mathrm{m}$, eje transapical: 4-6,5 $\mu \mathrm{m}$. Estrías: 18-20 $10 \mu \mathrm{m}$.

Ecologia: Especie ubiquitaria (Guiry et Guiry, 2020). Alcalófila, estuarina. Cosmopolita de aguas dulces a levemente salobres (Luchini et Verona, 1972).

Distribución geográfica: Buenos Aires, Chaco, Chubut, Córdoba, Misiones, La Pampa, San Luis, Río Negro y Santa Cruz (Tell, 1985; Vouilloud, 2003). En el NOA: Catamarca (Seeligmann et Maidana, 2003), Jujuy (Maidana et al., 1998), Santiago del Estero (Maidana et Herbst, 1989a) y Tucumán (Martínez De Marco, 2009; Taboada, 2017).

Material estudiado: Taboada 25.345 y 25.348 (LIL).

Cocconeis placentula var. euglypta (Ehrenberg) Grunow (Fig. 3F-G)

Valvas elípticas con extremos ampliamente redondeados. Valva con rafe: área axial lineal, angosta; área central circular, pequeña; rafe recto; estrías paralelas finamente areolas; anillo hialino submarginal delgado y poco conspicuo. Valva sin rafe: área axial angosta, lineal, área central ausente, estrías paralelas con areolas cortas transversalmente elongadas dispuestas formando líneas longitudinalmente irregulares. Dimensiones celulares: eje apical: $18-33 \mu \mathrm{m}$, eje transapical: $11-15 \mu \mathrm{m}$. Estrías: $10-18$ en $10 \mu \mathrm{m}$.

Ecología: Especie de agua dulce (Guiry et Guiry, 2020). Oligohalobia indiferente, alcalífila, mesoeutrófica, epífita, en aguas templadas, corriente indiferente. Cosmopolita (Luchini et Verona, 1972; Wolf, 1982).

Distribución geográfica: Antártida, Buenos Aires, Chaco, Chubut, Córdoba, Corrientes, Entre Ríos, La Pampa, Neuquén, Río Negro, San Luis y Tierra del Fuego (Luchini et Verona,1972; Tell, 1985; Vouilloud, 2003). En el NOA: Jujuy (Seeligmann et al., 2008; González Achem et al., 2014), Salta (Salusso, 1998) y Santiago del Estero (Maidana y Herbst, 1989a) y Tucumán (Mirande et Tracanna, 1995; Seeligmann, 1998; Martínez De Marco, 2009; Taboada et al., 2015, Taboada, 2017)

Material estudiado: Taboada $25.345 \mathrm{y}$ 25.346 (LIL).
Cocconeis placentula var. lineata (Ehrenberg) Van Heurck (Fig. 3H)

Se diferencia de la var. euglypta por el anillo hialino submarginal en la valva con rafe muy conspicuo, más ancho y con mayor grado de silicificación. Dimensiones celulares: eje apical: 24-48 $\mu \mathrm{m}$, eje transapical: $12-25 \mu \mathrm{m}$. Estrías: $15-25$ en 10 $\mu \mathrm{m}$.

Ecología: De agua dulce, cosmopolita (Luchini et Verona, 1972).

Distribución geográfica: Antártida, Buenos Aires, Chubut, Córdoba, Entre Ríos, La Pampa, Neuquén, Río Negro, Santa Cruz y Tierra del Fuego (Luchini et Verona, 1972; Tell, 1985; Vouilloud, 2003). En el NOA: Catamarca (Seeligmann et Maidana, 2003), Jujuy (Maidana et al., 1998; Seeligmann et al., 2008; González Achem et al., 2014), Salta (Moraña, 1998; Salusso, 1998) y Tucumán (Martínez De Marco, 2009; Taboada et al., 2015)

Material estudiado: Taboada 25.345 y 25.346 (LIL).

\section{Denticula elegans Kützing (Fig. 4I)}

Valvas lanceoladas con extremos cuneados, redondeados. Fíbulas prolongadas en costillas que atraviesan toda la valva y ligeramente capitadas en vista cingular. Estrías paralelas, notablemente areoladas. Dimensiones celulares: eje apical: 24-43 $\mu \mathrm{m}$, eje transapical: $6,5-8,5 \mu \mathrm{m}$. Fíbulas: 5-6 en $10 \mu \mathrm{m}$. Estrías: $15-18$ en $10 \mu \mathrm{m}$.

Ecologia: Agua dulce y salobre. Cosmopolita (Luchini et Verona, 1972).

Distribución geográfica: Buenos Aires, Chubut, Córdoba, San Luis, La Pampa, Río Negro (Vouilloud, 2003). En el NOA: Catamarca (Seeligmann et Maidana, 2003), Jujuy (Seeligmann et al., 2008; González Achem et al., 2014), Santiago del Estero (Maidana et Herbst, 1989a) y Tucumán (Martínez De Marco, 2009, Taboada, 2017). Material estudiado: Taboada 25.346 y 25.347 (LIL).

\section{Denticula kuetzingii Grunow (Fig. 4J-K)}

Valvas lanceoladas a lineal-lanceoladas, con extremos cuneados, redondeados a subrostrados. Fíbulas prolongadas en 

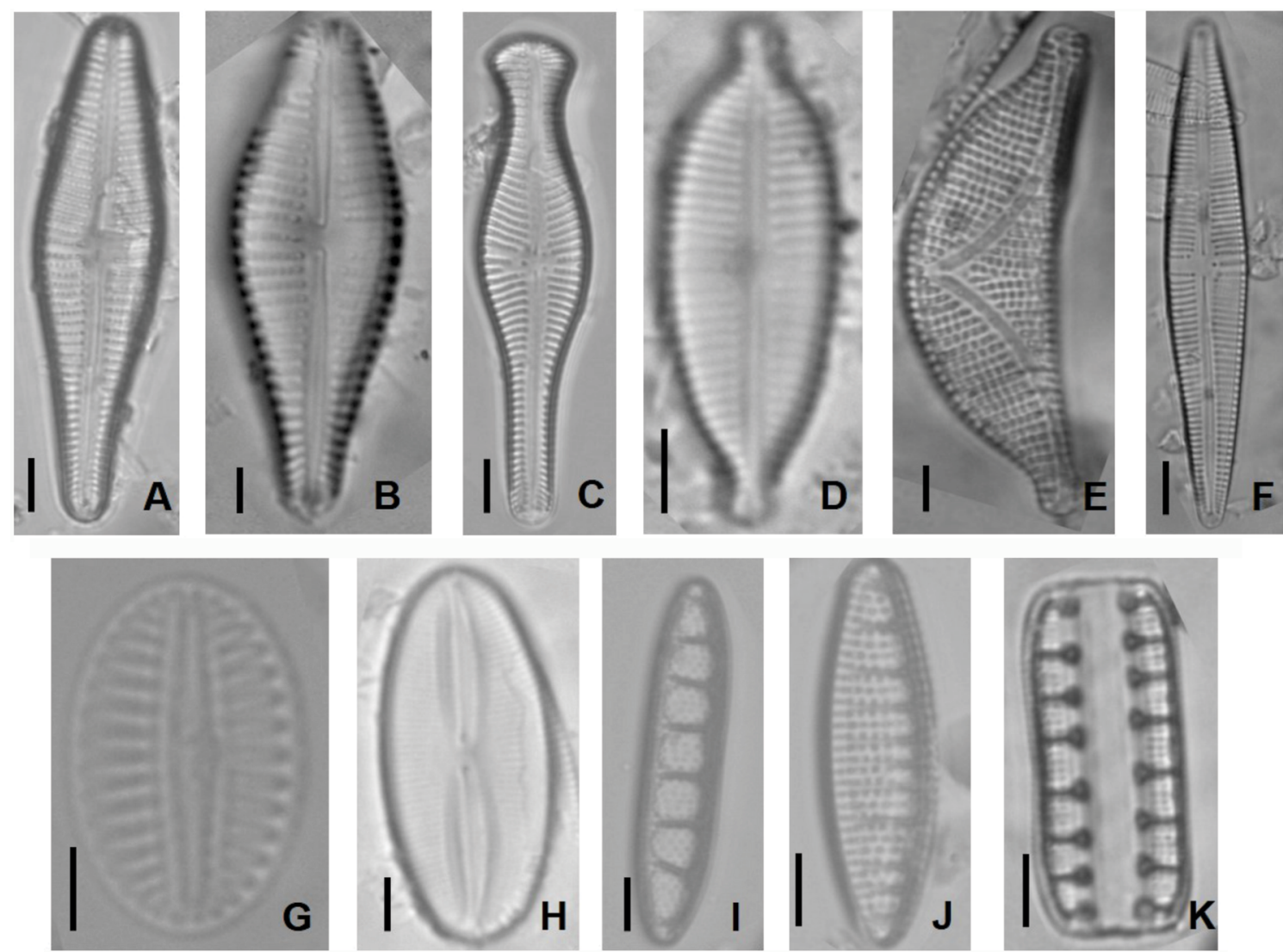

Fig. 4. Microfotografias de las diatomeas registradas en la laguna. A: Gomphonema affine B: G. affinopsis. C: G. capitatum. D: G. parvulum. E: Epithemia sorex. F: Gomphonema lippertii. G: Diploneis puella. H: Fallacia pygmaea. I: Denticula elegans. Denticula kuetzingii. J: Vista valvar. K: Vista cingular. La escala corresponde a $10 \mu \mathrm{m}$.

Fig. 4. Photomicrographs of diatoms recorded in the ponds. A: Gomphonema affine B: G. affinopsis. C: G. capitatum. D: G. parvulum. E: Epithemia sorex. F: Gomphonema lippertii. G: Diploneis puella. H: Fallacia pygmaea. I: Denticula elegans. Denticula kuetzingii. J: Valvar view. K: Cingular view. The scale corresponds to $10 \mu \mathrm{m}$.

costillas que se adelgazan progresivamente hacia el centro, no capitadas en vista cingular. Estrías paralelas, notablemente areoladas. Dimensiones celulares: eje apical 20-56 $\mu \mathrm{m}$, eje transapical 4,1-6,4 $\mu \mathrm{m}$. Fíbulas: 5-7 en $10 \mu \mathrm{m}$. Estrías: $14-16$ en $10 \mu \mathrm{m}$.

Ecologia: En aguas dulces y salobres, alcalinas, con contenido alto de $\mathrm{O}_{2}$, $\beta$-mesosaprobias y mesotróficas (Martínez de Fabricius, 1996).

Distribución geográfica: Antártida, Buenos Aires, Chubut, Córdoba, Entre Ríos, La Pampa, Neuquén, Río Negro, Santa Cruz, Tierra del Fuego (Luchini et Verona, 1972; Tell, 1985; Vouilloud, 2003). En el NOA: Catamarca (Seeligmann et Maidana, 2003) y Tucumán (Seeligmann, 1998; Isasmendi et al., 2002; Taboada, 2017).

Material estudiado: Taboada 25.345 y 25.346 (LIL).

Diploneis puella (Schumann) Cleve (Fig. 4G)

Valvas lineal-elíptica con extremos anchamente redondeados. Área central orbicular. Canales longitudinales angostos. Costillas paralelas. Dimensiones celulares: eje apical: 13-18 $\mu \mathrm{m}$, eje transapical: 9-13 $\mu \mathrm{m}$. Costillas: $11-12$ en $10 \mu \mathrm{m}$. Estrías: 12-13 en $10 \mu \mathrm{m}$.

Ecología: Especie de agua dulce (Guiry et Guiry, 2020). En ambientes con moderada 
a alta conductividad, $\beta$-mesosapróbica (Martínez de Fabricius, 1996).

Distribución geográfica: Buenos Aires, Córdoba, (Martínez de Fabricius, 1996; Vouilloud, 2003). En el NOA: Tucumán (Martínez De Marco, 2009; Taboada et al., 2016).

Material estudiado: Taboada 25.346(LIL).

\section{Epithemia sorex Kützing (Fig. 4E)}

Valvas dorsiventralmente arqueadas con el margen ventral levemente cóncavo y el dorsal convexo. Ápices rostrado-capitados. Canal rafidiano visible en todo su recorrido, con el nódulo central muy próximo al margen dorsal. Costillas separadas por 2-3 hileras de areolas. Dimensiones celulares: eje apical: 26-55 $\mu \mathrm{m}$, eje transapical: $9-12 \mu \mathrm{m}$. Costillas: 7-11 en 10 $\mu \mathrm{m}$. Estrías: $15-25$ en $10 \mu \mathrm{m}$.

Ecología: En aguas alcalinas, con contenido alto de $\mathrm{O}_{2}, \beta$-mesosaprobias, eutróficas (Martínez de Fabricius, 1996).

Distribución geográfica: Buenos Aires, Chubut, Córdoba, La Pampa, Neuquén y Tierra del Fuego. En el NOA: Jujuy (Maidana et al., 1998), Santiago del Estero (Maidana et Herbst, 1989a) y Tucumán (Isasmendi et al., 2002; Martínez De Marco, 2009).

Material estudiado: Taboada 25.345 y 25.346 (LIL).

Fallacia pygmaea (Kützing) Stickle \& D. G. Mann (Fig. 4H)

Las valvas son lanceoladas a elípticas, con extremos frecuentemente redondeados. El área axial es angosta y la central de forma y tamaño variables. Rafe filiforme, alojado en una costilla prominente enmarcada por un par de depresiones longitudinales, perforadas o no, que se visualizan al microscopio óptico (MO) como una zona hialina en forma de H. Las estrías son uniseriadas, con areolas de contorno variable. Dimensiones celulares: eje apical: 20-38 $\mu \mathrm{m}$, eje transapical: 8-12 $\mu \mathrm{m}$. Estrías: 15-20 en $10 \mu \mathrm{m}$.

Ecología: De agua dulce (Guiry et Guiry, 2020). Especie $\alpha-\beta$-mesosapróbica, mesooxibionte. Cosmopolita de aguas dulces y salobres, con alta conductividad y, a veces en sistemas poluídos (Luchini et Verona, 1972; Krammer et Lange-Bertalot, 1986).
Distribución geográfica: Buenos Aires, Chaco, Córdoba y La Pampa (Vouilloud, 2003). En el NOA: Jujuy (González Achem et al., 2014) y Tucumán (Isasmendi et al., 2002; Taboada et al., 2015; 2016; Taboada, 2017).

Material estudiado: Taboada 25.345 y 25.346 (LIL).

\section{Gomphonema affine Kützing (Fig. 4A)}

Valvas lanceoladas, con extremos redondeados. Área axial angosta. Área central con un estigma. Estrías radiales en toda la valva. Dimensiones celulares: eje apical: 43-76 $\mu \mathrm{m}$, eje transapical: 9-12 $\mu \mathrm{m}$. Estrías: 9-13 en $10 \mu \mathrm{m}$.

Ecología: Oligohalobia indiferente, alcalífila, litoral, tolera amplios rangos de conductividad en agua dulce (Patrick et Reimer, 1975).

Distribución geográfica: Buenos Aires, Chaco, Córdoba, Corrientes y Entre Ríos (Vouilloud, 2003). En el NOA: Catamarca (Seeligmann et Maidana, 2003) y Tucumán (Maidana et Herbst, 1989b; Taboada et al., 2016; Taboada, 2017).

Material estudiado: Taboada $25.345 \mathrm{y}$ 25.346 (LIL).

Gomphonema affinopsis Metzeltin \& García-Rodríguez (Fig. 4B)

Valvas elíptico-lanceoladas con extremos rostrados y redondeados, área axial angosta, levemente expandida en el centro. Con un solo estigma elongado en el área central. El rafe es lateral y débilmente ondulado. Los extremos del rafe proximal externo están ligeramente dilatados. Dimensiones celulares: eje apical: 35-48 $\mu \mathrm{m}$, eje transapical: $11-13 \mu \mathrm{m}$. Estrías: 9-10 en $10 \mu \mathrm{m}$.

Ecología: Agua dulce y salobre (Guiry et Guiry, 2020).

Distribución geográfica: Tucumán (Taboada, 2017).

Material estudiado: Taboada 25.345 (LIL).

Gomphonema capitatum Ehrenberg (Fig. 4C)

Valva claviforme, notablemente asimétrica, dilatada en la zona media; ápice de ancho similar a la zona central con 
extremo redondeado; base angosta con extremo redondeado. Área axial sinuosa, área central asimétrica, con la estría central más larga que las dos adyacentes. Un estigmoide en el extremo de una de las estrías más largas, cerca del nódulo central. Rafe lateral, con los poros centrales poco expandidos. Estrías radiales en la zona media de la valva, levemente radiales hacia los extremos. Areolas a veces distinguibles con MO. Dimensiones celulares: eje apical: $32-44 \mu \mathrm{m}$; eje transapical: 8-11 $\mu \mathrm{m}$. Estrías: 10-15 en $10 \mu \mathrm{m}$.

Ecología: De agua dulce/salobre (Guiry et Guiry, 2020).

Distribución geográfica: Buenos Aires, Córdoba y Tierra del Fuego (Vouilloud 2003). En el NOA: Salta (Moraña, 1998; Salusso, 1998) y Tucumán (Tracanna et Martínez de Marco, 1997; Seeligmann et al., 1998; Isasmendi et al., 2002; Taboada et al., 2015; 2016; Taboada, 2017).

Material estudiado: Taboada $25.345 \mathrm{y}$ 25.346 (LIL).

\section{Gomphonema lippertii Reichardt \& Lange-} Bertalot (Fig. 4F)

Valvas lanceoladas a rómbico lanceoladas, ligeramente cimbeloides; ápice angosto, con extremo redondeado. Área axial angosta; área central delimitada por estrías cortas; con estigma ubicado cerca del nódulo central, algo separado de la estría media. Rafe lateral, poco curvado a casi recto. Estrías ligeramente radiales y en los extremos más densamente dispuestas. Aréolas difíciles de distinguir al MO. Dimensiones celulares: eje apical: 40,455,8 $\mu \mathrm{m}$; eje transapical: $10-12 \mu \mathrm{m}$. Estrías: 10-11 en $10 \mu \mathrm{m}$.

Ecología: En ambientes con alto contenido de carbonatos y elevada conductividad eléctrica (Reichardt, 1999).

Distribución geográfica: Tucumán (Nieva et al., 2019)

Material estudiado: Taboada 25.346(LIL).

\section{Gomphonema parvulum (Kützing) Kützing (Fig. 4D)}

Valvas muy variables en cuanto al contorno, desde anchamente lanceoladas hasta angostamente elípticas, con el ápice siempre diferenciable del cuerpo principal y la base subrostrada. Área axial angosta; área central asimétrica delimitada por el acortamiento de una estría central, un estigmoide en el extremo de la estría opuesta. Estrías levemente radiales a paralelas en la zona media de la valva, suavemente radiales hacia los extremos. Areolas no distinguibles en MO. Dimensiones celulares: eje apical: $25-40$ $\mu \mathrm{m}$; eje transapical: 8-10 $\mu \mathrm{m}$. Estrías: 9-15 en $10 \mu \mathrm{m}$.

Ecología: Agua dulce (Guiry et Guiry, 2020). De pH indiferente, eurioxibionte (saprófila), aerófila, oligohalobiaindiferente, $\mathrm{pH}$ indiferente, mesoeutrófica, de aguas cálidas, templadas, reófila $\alpha$ - mesopolisaprobia. Cosmopolita en agua dulce y salobre con moderada a alta conductividad, ubicuitaria (Luchini et Verona, 1972; Martínez de Fabricius, 1996).

Distribución geográfica: Buenos Aires, Chubut, Córdoba, Corrientes, Entre Ríos, La Pampa, Misiones, Río Negro y San Luis (Tell, 1985; Vouilloud, 2003). En el NOA: Catamarca (Seeligmann et Maidana, 2003), Jujuy (Maidana et al., 1998; Gónzalez Achem et al., 2014), Salta (Moraña, 1998; Salusso, 1998) y Tucumán (Seeligmann, 1998; Isasmendi et al., 2002; Mirande et Tracanna, 2003; Martínez De Marco, 2009; Taboada et al., 2015; 2016; Taboada, 2017).

Material estudiado: Taboada 25.345 y 25.346 (LIL).

Luticola kotschyi (Grunow) Taylor, Harding \& Archibald (Fig. 5G)

Valvas elípticas de ápices más o menos redondeados. Área axial recta. Área central muy amplia con estigma. Rafe recto, con extremos proximales curvados en la misma dirección y los distales a modo de signo de interrogación. Estrías radiales y areolas visibles al MO. Dimensiones celulares: eje apical: $10-16 \mu \mathrm{m}$, eje transapical: $5,5-7,8 \mu \mathrm{m}$. Estrías: $18-20$ en $10 \mu \mathrm{m}$.

Ecología: Aerófila, epífita, epilítica y fitoplanctónica. En agua dulce a levemente salobre, en moderada a alta conductividad, $\mathrm{pH}$ neutro a alcalino, $\beta$-mesosapróbica. Cosmopolita de aguas dulces (Guiry et Guiry, 2020; Luchini et Verona, 1972; Martínez de Fabricius, 1996). 

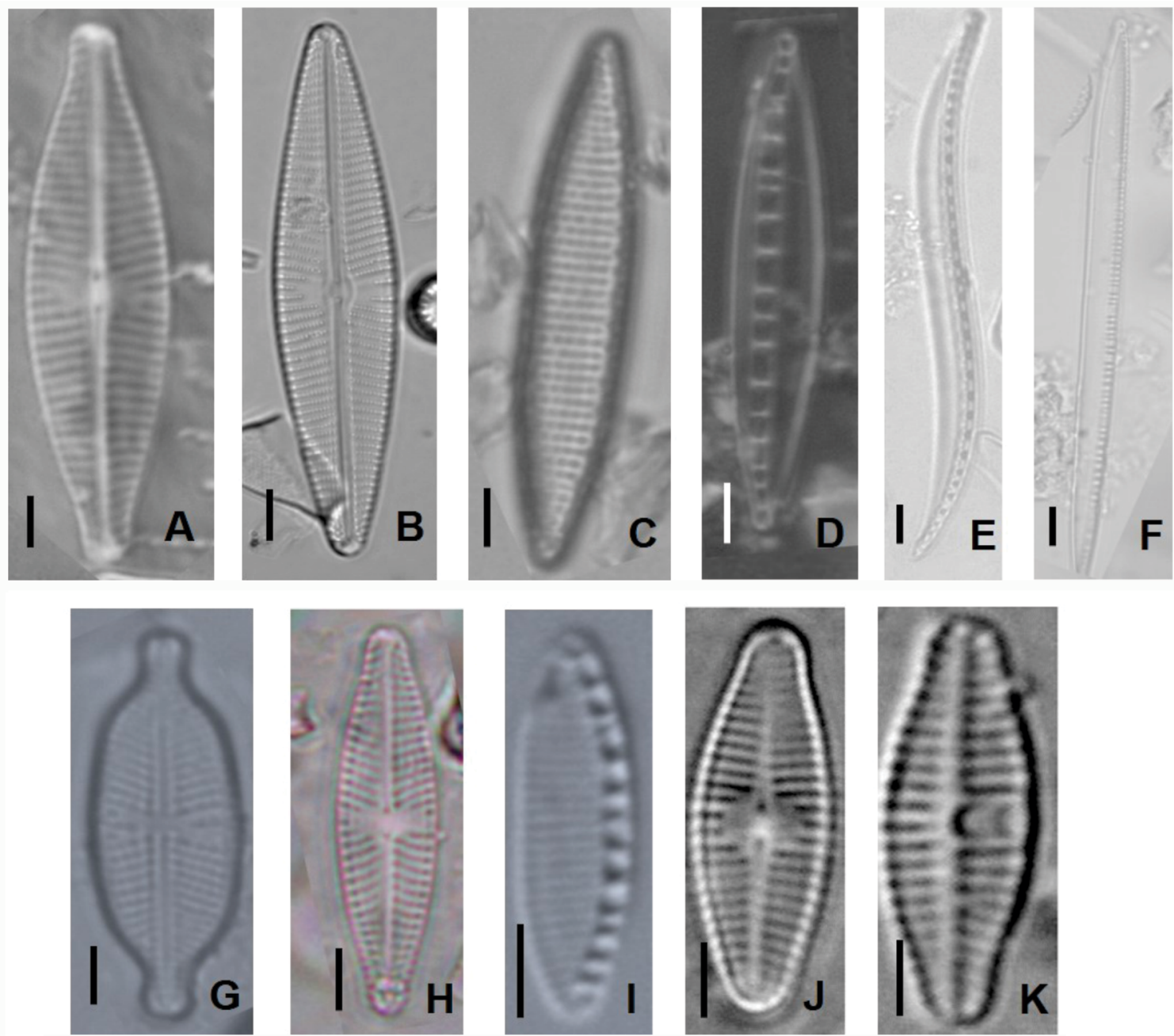

Fig. 5. Microfotografias de las diatomeas registradas en la laguna. A: Navicula cryptocephala. B: N. peregrina. C: Nitzschia amphibia. D: N. dissipata. E: N. sigma. F: N. linearis. G: Luticola kotschyi. H: Navicula veneta. I: Nitzschia insconspicua. Planothidium frecuentissimum. J: Valva con rafe. K: Valva sin rafe. La escala corresponde a $10 \mu \mathrm{m}$.

Fig. 5: Photomicrographs of diatoms recorded in the ponds. A: Navicula cryptocephala. B: N. peregrina. C: Nitzschia amphibia. D: N. dissipata. E: N. sigma. F: N. linearis. G: Luticola kotschyi. H: Navicula veneta. I: Nitzschia insconspicua. Planothidium frecuentissimum. J: Valve with raphe. K: Valve without raphe. The scale corresponds to $10 \mu \mathrm{m}$.

Distribución geográfica: Buenos Aires, Córdoba, Misiones y San Luis (Vouilloud, 2003). En el NOA: Catamarca (Seeligmann et Maidana, 2003) y Tucumán (Martínez De Marco, 2009; Taboada, 2017).

Material estudiado: Taboada 25.345 (LIL).

Navicula cryptocephala Kützing (Fig. 5A)

Valvas lanceoladas a angostamente lanceoladas con extremos desde subrostrados hasta subcapitados. Área axial angosta a muy angosta; área central orbicular a transversalmente elíptica, ligeramente asimétrica. Rafe filiforme. Estrías fuertemente radiales en la zona media de la valva, ligeramente convergentes hacia los extremos. Dimensiones celulares: eje apical: 25,8-27 $\mu \mathrm{m}$, eje transapical: $6,5-7,3 \mu \mathrm{m}$. Estrías: 16-20 en $10 \mu \mathrm{m}$.

Ecología: Especie de agua dulce/ salobre (Guiry et Guiry, 2020). Bentónica, 
en aguas dulces a ligeramente salobres, circumneutrales, con contenido relativamente alto de $\mathrm{O}_{2}, \alpha$-mesosaprobias. Esta especie tiene un amplio rango de tolerancia en cuanto al $\mathrm{pH}$, contenido de nutrientes y electrolitos (Luchini et Verona, 1972; Martínez de Fabricius, 1996).

Distribución geográfica: Buenos Aires, Chaco, Córdoba, Corrientes, Entre Ríos, La Pampa, Mendoza, Misiones, Neuquén y Río Negro (Vouilloud, 2003). En el NOA: Jujuy (Maidana et al., 1998; González Achem et al., 2014), Salta (Moraña, 1998) y Tucumán (Martínez De Marco, 2009; Taboada, 2017).

Material estudiado: Taboada $25.345 \mathrm{y}$ 25.346 (LIL).

Navicula peregrina (Ehrenberg) Kützing (Fig. 5B)

Valvas lanceoladas con extremos redondeados, a veces ligeramente proyectados. Área axial angosta, linear; área central transversal, de contorno variable. Rafe lateral, con los poros centrales notables, curvados en forma de gancho. Estrías notablemente lineoladas, radiales en la zona media de la valva, convergentes hacia los extremos. Dimensiones celulares: eje apical: 70-112 $\mu \mathrm{m}$, eje transapical:15-20 $\mu \mathrm{m}$. Estrías: 6-7 en $10 \mu \mathrm{m}$.

Ecología: Especie de agua salobre (Guiry et Guiry, 2020). En ambientes continentales con elevada concentración salina o alta conductividad (Martínez de Fabricius, 1996).

Distribución geográfica: Antártida, Buenos Aires, Chaco, Chubut, Córdoba, Corrientes, Entre Ríos, La Pampa, Neuquén, San Luis, Santa Fe y Tierra del Fuego (Luchini et Verona, 1972; Vouilloud, 2003). En el NOA: Catamarca (Seeligmann et Maidana, 2003; Seeligmann et Maidana, 2013), Jujuy (Maidana et al., 1998; Maidana et al., 2011; González Achem et al., 2014), Salta (Vouilloud, 2003) y Tucumán (Tracanna et Seeligmann, 1992; Mirande et Tracanna, 1995; Tracanna et Martínez De Marco, 1997; Seeligmann, 1998; Mirande, 2001; Isasmendi et al., 2002; Martínez De Marco, 2009; Taboada et al., 2015; 2016; Taboada, 2017).

Material estudiado: Taboada $25.345 \mathrm{y}$ 25.346 (LIL).
Navicula veneta Kützing (Fig. $5 \mathrm{H}$ )

Valvas lanceoladas a lineal-lanceoladas con extremos subrostrados a rostrados. Área axial angosta, lineal; área central transversal, casi simétrica. Rafe filiforme. Estrías moderadamente radiales en la zona media de la valva, convergentes en los extremos. Dimensiones celulares: eje apical: $18-28 \mu \mathrm{m}$, eje transapical: $4,5-6 \mu \mathrm{m}$. Estrías: $14-16$ en 10 $\mu \mathrm{m}$.

Ecología: Especie de agua salobre (Guiry et Guiry, 2020). En aguas alcalinas, con contenido relativamente alto de $\mathrm{O}_{2}, \alpha$-mesopolisaprobias, eutróficas (Krammer et Lange-Bertalot, 1986).

Distribución geográfica: Buenos Aires, Chubut, Córdoba, Entre Ríos y La Pampa, (Vouilloud, 2003). En el NOA: Jujuy (Maidana et al., 1998; González Achem et al., 2014), Salta (Salusso, 2005) y Tucumán (Martínez De Marco, 2009).

Material estudiado: Taboada 25.346(LIL).

\section{Nitzschia amphibia Grunow (Fig. 5C)}

Valvas lanceoladas con extremos cuneoredondeados. Carina marginal. Estrías con poroides notables. Dimensiones celulares: eje apical:14,9 -26,4 $\mu \mathrm{m}$, eje transapical: $2,9-4,8$ $\mu \mathrm{m}$. Fíbulas: $15-18$ en $10 \mu \mathrm{m}$. Estrías: 14-16 en $10 \mu \mathrm{m}$.

Ecología: Especie de agua dulce (Guiry et Guiry, 2020). Oligohalobia indiferente, alcalófila, mesooxibionte, euriplástica. Aguas dulces y levemente salobres (Luchini et Verona, 1972).

Distribución geográfica: Buenos Aires, Chaco, Chubut, Córdoba, Corrientes, Entre Ríos, La Pampa, Misiones, Neuquén, Río Negro, Santa Cruz, Santa Fe y Tierra del Fuego (Luchini et Verona,1972; Tell, 1985; Vouilloud, 2003). En el NOA: Catamarca (Seeligmann et Maidana, 2003), Jujuy (Maidana et al., 1998), Salta (Salusso, 1998) y Tucumán (Isasmendi et al., 2002; Taboada et al., 2015; Taboada, 2017).

Material estudiado: Taboada 25.345 y 25.346 (LIL).

Nitzschia dissipata (Kützing) Grunow (Fig. 5D)

Valvas lineal-lanceoladas con extremos subrostrados a rostrados. Canal rafidiano subcentral, prominente; fíbulas irregularmente 
distanciadas, alargadas, demarcando interespacios irregulares, las dos centrales no están más separadas entre sí que el resto. Estrías indistinguibles con MO. Dimensiones celulares: eje apical: 30,2-75 $\mu \mathrm{m}$; eje transapical: 3-5 $\mu \mathrm{m}$. Fíbulas: 6-11 en $10 \mu \mathrm{m}$.

Ecología: En aguas dulces a levemente salobres, alcalinas, con contenido relativamente alto de $\mathrm{O}_{2}, \beta$-mesosaprobias, meso-eutróficas (Luchini et Verona, 1972).

Distribución geográfica: Buenos Aires, Córdoba, La Pampa, Neuquén y Río Negro (Vouilloud, 2003). En el NOA: Catamarca (Seeligmann et Maidana, 2003), Jujuy (Maidana et al., 1998), Santiago del Estero (Maidana et Herbst, 1989a) y Tucumán (Isasmendi, 2016; Taboada, 2017).

Material estudiado: Taboada $25.345 \mathrm{y}$ 25.346 (LIL).

\section{*Nitzschia inconspicua Grunow (Fig. 5I)}

Valvas pequeñas, elíptico-lanceoladas con extremos redondeados a ligeramente aguzados. Canal rafidiano excéntrico; rafe interrumpido en la zona media; fíbulas conspicuas, irregularmente espaciadas. Estrías casi imperceptibles con MO; en la zona de la carina hay 2 poroides diminutos en el extremo de cada estría. Dimensiones celulares: eje apical: 5-6,4 $\mu \mathrm{m}$, eje transapical: $3-4,2 \mu \mathrm{m}$. Fíbulas: 11 en $10 \mu \mathrm{m}$. 20-26. Estrías: en 10 $\mu \mathrm{m}$.

Ecología: Especie de agua dulce (Guiry et Guiry, 2020). Cosmopolita en aguas salobres y dulces, alcalinas, con contenido moderado de $\mathrm{O}_{2}, \alpha$-mesosaprobias, eutróficas (Luchini et Verona, 1972).

Distribución geográfica: Buenos Aires, Córdoba, La Pampa y Río Negro (Vouilloud, 2003). En el NOA: Jujuy (Maidana et al., 1998). Primera cita para Tucumán.

Material estudiado: Taboada 25.345 y 25.346 (LIL).

\section{Nitzschia linearis Smith (Fig. 5F)}

Valvas lineales, a veces con una constricción en la zona media y extremos rostrados. Canal rafidiano submarginal, con una escotadura en el centro, no siempre fácil de distinguir; fíbulas lineales y angostas, las dos centrales están más distanciadas entre sí que el resto. Estrías apenas perceptibles con MO. Dimensiones celulares: eje apical: 95-110 $\mu \mathrm{m}$, eje transapical: 5-7 $\mu \mathrm{m}$. Fíbulas: 8-10 en $10 \mu \mathrm{m}$. Estrías: 26-28 en $10 \mu \mathrm{m}$.

Ecología: Especie de agua dulce (Guiry et Guiry, 2020). Gran amplitud ecológica, suelen habitar aguas de $\mathrm{pH}$ casi neutro, bien oxigenadas, moderada a altamente mineralizadas, ricas en materia orgánica y nutrientes (Martínez de Fabricius, 1996).

Distribución geográfica: Buenos Aires, Chaco, Chubut, Córdoba, Corrientes, La Pampa, Misiones, Mendoza, Neuquén, Río Negro, Santa Cruz y Tierra del Fuego (Luchini et Verona, 1972; Tell, 1985; Vouilloud, 2003). En el NOA: Catamarca (Seeligmann et Maidana, 2003), Jujuy (Maidana et al., 1998), Salta (Salusso, 1998) y Tucumán (Seeligmann, 1998; Mirande, 2001; Isasmendi et al., 2002; Mirande et Tracanna, 2003; Martínez De Marco, 2009; Taboada et al., 2015).

Material estudiado: Taboada 25.345 y 25.346 (LIL).

\section{Nitzschia sigma (Kützing) Smith (Fig. 5E)}

Valva sigmoidea con extremos agudos. Canal rafidiano marginal; fíbulas equidistantes. Estrías con areolas transapicalmente elongadas e irregularmente espaciadas, de modo tal que al MO se ven como líneas longitudinales irregulares. Dimensiones celulares: eje apical: 75-86 $\mu \mathrm{m}$, eje transapical: 4,5-6 $\mu \mathrm{m}$. Fíbulas: 10-11 en $10 \mu \mathrm{m}$. Estrías: $23-25$ en $10 \mu \mathrm{m}$.

Ecología: Especie de agua salobre (Guiry et Guiry, 2020). En aguas salobres, alcalinas, con contenido moderado de $\mathrm{O}_{2} \alpha$-mesosaprobias, eutróficas (Martínez de Fabricius, 1996).

Distribución geográfica: Buenos Aires, Chaco, Chubut, Córdoba, Corrientes, Entre Ríos, La Pampa, Río Negro y Tierra del Fuego (Vouilloud, 2003). En el NOA: Jujuy (Maidana et al., 1998; González Achem et al., 2014) y Tucumán (Isasmendi et al., 2002; Taboada, 2017).

Material estudiado: Taboada 25.345 y 25.346 (LIL).

Planothidium frequentissimum (LangeBertalot) Lange-Bertalot (Fig. 5J-K) Valvas elípticas, elíptico-lanceoladas a 
lanceoladas con extremos redondeados a rostrados. Valva con rafe: área axial angosta, lineal o lanceolada; área central rectangular o elíptica; rafe recto con las fisuras proximales expandidas y distales curvadas. Valva sin rafe con un cavum evidente. Estrías radiales en ambas valvas. Dimensiones celulares: eje apical: 14-20 $\mu \mathrm{m}$, eje transapical: 6-8 $\mu \mathrm{m}$. Estrías: $13-14$ en $10 \mu \mathrm{m}$.

Ecología: Especie de agua dulce y salobre (Guiry et Guiry, 2020).

Distribución geográfica: Buenos Aires, Córdoba, Neuquén y Tierra del Fuego (Vouilloud, 2003). En el NOA: Catamarca (Seeligmann et Maidana, 2003), Jujuy (Seeligmann et al., 2008; Gónzalez Achem et al., 2014) y Tucumán (Martínez De Marco, 2009; Taboada et al., 2016; Taboada, 2017).

Material estudiado: Taboada $25.345 \mathrm{y}$ 25.346 (LIL).

\section{Rhopalodia gibba (Ehrenberg) O. Müller (Fig. 6A)}

Valvas ligeramente arqueadas, con margen dorsal dilatado en su parte central, con o sin hendidura media y el margen ventral recto. Extremos curvados hacia el lado ventral. Rafe sin nódulos marcados; canal rafidiano dorsal. Costillas paralelas en la zona media de la valva, ligeramente radiales hacia los extremos, con 2-3 estrías entre ellas. Dimensiones celulares: eje apical: 35,7-84,5 $\mu \mathrm{m}$, eje transapical: $6,7-9,4 \mu \mathrm{m}$. Costillas: $6-7$ en $10 \mu \mathrm{m}$. Estrías: 14-16 en $10 \mu \mathrm{m}$.

Ecología: Especie de agua dulce (Guiry et Guiry, 2020). Epífita y fitoplanctónica en aguas dulces a ligeramente salobre, alcalinas, con moderado contenido de $\mathrm{O}_{2}, \beta$-mesosaprobias, eutróficas (Martínez de Fabricius, 1996).

Distribución geográfica: Buenos Aires, Chaco, Chubut, Córdoba, Corrientes, Entre Ríos, La Pampa, Mendoza, Neuquén, San Luis, Río Negro y Tierra del Fuego (Vouilloud, 2003). En el NOA: Catamarca (Seeligmann et Maidana, 2003), Jujuy (Maidana et al., 1998; Seeligmann et al., 2008; González Achem et al., 2014), Salta (Salusso, 1998) y Tucumán (Mirande et Tracanna, 1995; Tracanna et Martínez De Marco, 1997; Seeligmann, 1998; Isasmendi et al., 2002; Martínez De Marco, 2009; Taboada et al.; 2016; Taboada, 2017)
Material estudiado: Taboada 25.345 y 25.346 (LIL).

*Stauroneis anceps Ehrenberg (Fig. 6B)

Valvas lanceoladas con ápices capitados, área axial lineal, estauro relativamente ancho de forma más o menos rectangular. Rafe recto y filiforme. Estrías radiales formadas por pequeñas y delicadas areolas. Dimensiones celulares: eje apical: 43,5-56,2 $\mu \mathrm{m}$, eje transapical: 7,5-8,9 $\mu \mathrm{m}$. Estrías: $20-22$ en 10 $\mu \mathrm{m}$.

Ecología: Especie de agua dulce (Guiry et Guiry, 2020).

Distribución geográfica: Buenos Aires, Corrientes, La Pampa, Neuquén, Río Negro, Santa Cruz y Tierra del Fuego (Vouilloud, 2003). En el NOA: Jujuy (Maidana et al., 1998). Primer registro para Tucumán.

Material estudiado: Taboada 25.345 (LIL).

Stauroneis phoenicenteron (Hustedt) Krammer \& Lange-Bertalot (Fig. 6C)

Valvas lanceoladas con extremos subrostrados. Área axial ancha, levemente constricta en los extremos. Área central formando un estauro de forma rectangular levemente más ensanchada hacia los márgenes. Rafe recto. Estrías radiales, poroides visibles a MO. Dimensiones celulares: eje apical: 92,4 -145,5 $\mu \mathrm{m}$, eje transapical: 19,5- 26,7 $\mu \mathrm{m}$. Estrías: $15-18$ en $10 \mu \mathrm{m}$.

Ecología: Especie de agua dulce (Guiry et Guiry, 2020).

Distribución geográfica: Buenos Aires, Chaco, Córdoba, Corrientes, La Pampa, Neuquén, Santa Cruz, Santa Fe, San Luis y Tierra del Fuego (Vouilloud, 2003). En el NOA: Catamarca (Maidana et Seeligmann 2006), Jujuy (Seeligmann, 2008) y Tucumán (Nieva et al., 2019)

Material estudiado: Taboada 25.345 (LIL).

\section{Surirella ovalis Brèbisson (Fig. 6D)}

Valva heteropolar con extremos cuneados. Canales alares ausentes; fíbulas prolongadas en costillas cortas. Superficie valvar ondulada longitudinalmente. Estrías conspicuas en toda la valva. Dimensiones celulares: eje apical: $25-33$ $\mu \mathrm{m}$, eje transapical: $28-31 \mu \mathrm{m}$. Fíbulas: 4 en 10 $\mu \mathrm{m}$. Estrías: 15 en $10 \mu \mathrm{m}$. 

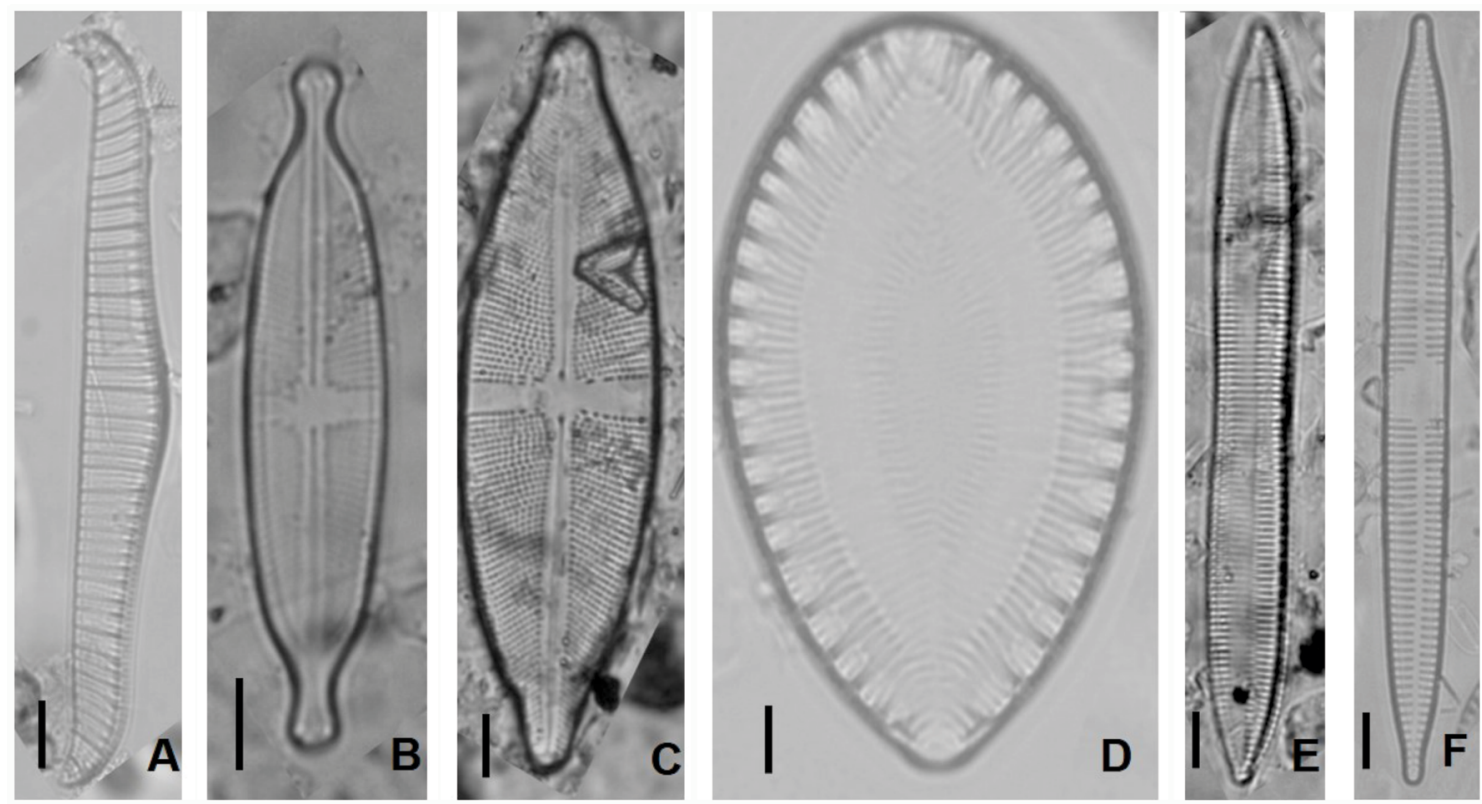

Fig. 6. Microfotografías de las diatomeas registradas en la laguna. A: Rhopalodia gibba B: Stauroneis anceps. C: S. phoenicenteron D: Surirella ovalis. E: Tryblionella hungarica. F: Ulnaria ulna. La escala corresponde a $10 \mu \mathrm{m}$. Fig. 6. Photomicrographs of diatoms recorded in the ponds. A: Rhopalodia gibba B: Stauroneis anceps. C: S. phoenicenteron D: Surirella ovalis. E: Tryblionella hungarica. F: Ulnaria ulna. The scale corresponds to $10 \mu \mathrm{m}$.

Ecología: Bentónica, en aguas salobres a dulces, alcalina, con contenido bajo de $\mathrm{O}_{2}$ $\alpha$-mesosaprobias, eutróficas (Guiry et Guiry, 2020, Luchini et Verona, 1972).

Distribución geográfica: Buenos Aires, Chaco, Chubut, Córdoba, La Pampa, Misiones, Neuquén, Río Negro, Santa Cruz y Tierra del Fuego (Vouilloud, 2003). En el NOA: Catamarca (Seeligmann et Maidana, 2003), Jujuy (Maidana et al., 1998; González Achem et al., 2014), Salta (Salusso, 1998), Santiago del Estero (Maidana et Herbst, 1989a) y Tucumán (Tracanna et Martínez De Marco, 1997; Seeligmann, 1998; Isasmendi et al., 2002; Martínez De Marco, 2009; Taboada et al., 2016).

Material estudiado: Taboada 25.345 y 25.346 (LIL).

\section{Tryblionella hungarica (Grunow) Frenguelli (Fig. 6E)}

Valvas lineales, levemente cóncavas en la zona media, con extremos cuneados, a veces subrostrados. Carina marginal; fíbulas distribuidas regular o regularmente; siempre las dos centrales un poco más distanciadas que el resto. Estrías más numerosas que las fíbulas e interrumpidas en el centro de la valva por una zona hialina que ocupa aproximadamente 1/3 del ancho valvar. Dimensiones celulares: eje apical: 48-75 $\mu \mathrm{m}$, eje transapical: 7-9 $\mu \mathrm{m}$. Fíbulas: $8-11$ en $10 \mu \mathrm{m}$. Estrías: $15-17$ en 10 $\mu \mathrm{m}$.

Ecología: Especie de agua salobre (Guiry et Guiry, 2020). Planctónica, de aguas dulces y levemente salobres, alcalinas y eutróficas, especie $\alpha$-mesosaprobia.

Distribución geográfica: Buenos Aires, Chaco, Córdoba, Entre Ríos y La Pampa (Vouilloud, 2003). En el NOA: Catamarca (Seeligmann et Maidana, 2003), Jujuy (Maidana et al., 1998; González Achem et al., 2014) y Tucumán (Taboada et al., 2016; Taboada, 2017).

Material estudiado: Taboada 25.345 y 25.346 (LIL).

Ulnaria ulna (Nitzsch) Compère (Fig. 6F)

Valvas lineales a lineal-lanceoladas, atenuadas gradualmente en los extremos, que 
son rostrados o subacapitados. Área axial linear, angosta; área central rectangular, generalmente más larga que ancha, totalmente sin estrías o con estrías solo marginales. Estrías paralelas, opuestas. Dimensiones celulares: eje apical: 115-130 $\mu \mathrm{m}$, eje transapical: $6,5-$ 7,5 $\mu \mathrm{m}$. Estrías: 9 en $10 \mu \mathrm{m}$.

Ecología: Especie de agua dulce (Guiry et Guiry, 2020). En aguas dulces a salobres, alcalinas, con contenido moderado de $\mathrm{O}_{2}$, $\alpha$-meso-polisaprobias, oligo-eutróficas (Martínez de Fabricius, 1996).

Distribución geográfica: Antártida, Buenos Aires, Chaco, Chubut, Córdoba, Corrientes, Entre Ríos, Formosa, La Pampa, Mendoza, Misiones, Neuquén, Río Negro, Santa Fe y Tierra del Fuego (Vouilloud, 2003). En el NOA: Catamarca (Seeligmann et Maidana, 2003), Jujuy (González Achem et al., 2014), Salta (Moraña, 1998; Salusso, 1998), Santiago del Estero (Seeligmann et Maidana, 2003) y Tucumán (Mirande et Tracanna, 1995; Tracanna et Martínez De Marco, 1997; Seeligmann, 1998; Mirande et al., 2000; Martínez De Marco et Tracanna, 2001; Isasmendi et al., 2002; Mirande et Tracanna, 2003; Martínez De Marco, 2009; Seeligmann et Tracanna, 2009; Taboada et al., 2015; Taboada, 2017).

Material estudiado: Taboada $25.345 \mathrm{y}$ 25.346 (LIL).

\section{Discusión y Conclusiones}

En los últimos años la atención de los investigadores se centró en la disminución de la biodiversidad en ambientes terrestres $\mathrm{y}$ solo muy recientemente ha aumentado la preocupación por la pérdida de especies en sistemas acuáticos. Uno de los ecosistemas más amenazados en el NOA son los de agua dulce que albergan una biodiversidad única y se han registrado elevadas densidades de su biota (Nieto Peñalver et al., 2017, Schröter et al., 2005). La escasez de inventarios taxonómicos impide que se tenga claridad respecto del real efecto del cambio climático sobre la diversidad. La hipótesis de la disminución de la biodiversidad debida a cambios ambientales de origen natural o antrópico puede ser analizada a través de un estudio de la evolución paleoambiental en una región determinada, para lo cual es imprescindible el conocimiento de la diversidad presente y pasada (Mann, 1999).

La región de Yungas resulta interesante para la concreción de múltiples estudios, no sólo por su geografía privilegiada sino también por la enorme riqueza, variedad de ambientes y el gran número de sistemas lóticos y leníticos que posee (Quiroga et Grau, 2020). A pesar de ello, si se compara con otras regiones del mundo, ha sido poco estudiada desde el punto de vista de las taxocenosis algales.

Los endicamientos naturales generan, por un lado, el cese de aporte de agua de un curso de orden inferior a uno de orden superior, y por otro, un posible escenario de generación de un flujo de detritos de grandes proporciones si se produce el colapso poniendo en riesgo obras de infraestructura que se encontrasen aguas abajo. Este tipo de endicamientos, ya han sido registrados para el Holoceno (últimos 10.000 años) dentro de la cuenca del río Potrerillos (Moyano, 2006) a partir del hallazgo de fósiles de sedimentación relíctica de ambientes lacustres en afloramientos cuaternarios. Las lagunas de altura y formadas por deslizamientos de montañas son escasas, de distribución heterogénea y constituyen ecosistemas frágiles, vulnerables a la intervención humana y a efectos naturales. Los sedimentos lacustres preservados en ellos, actuales o fósiles relícticos, constituyen importantes registros capaces de revelar la historia paleoambiental de esas áreas. La comparación entre los eventos actuales y pasados puede brindar información acerca del comportamiento que tiene este tipo de procesos desde el punto de vista hidrológico y paleoclimático.

En este primer relevamiento ficológico de la laguna del Siambón se registraron un total de 39 especies, de las cuales 32 corresponden a diatomeas, apreciando así su elevada riqueza, aún en un número reducido de muestras y considerando solamente al fitoplancton. Las diatomeas se destacan por su amplia variedad de estrategias adaptativas, su corto ciclo de vida, tamaño pequeño, rapidez y eficacia de colonización. Se suma a ello la elevada eficiencia fotosintética, alto contenido de 
clorofila y bajo umbral de saturación de luz (Round et al., 1990; Moreno Montoya et Aguirre, 2013), características que les permite adaptarse a una gran variedad de ambientes, lo que se vio reflejado en este trabajo, en el cual fueron mucho más frecuentes que el resto de los grupos ficológicos. Las especies con las frecuencias más altas de aparición pertenecen a los taxones Amphipleura lindheimeri, Cocconeis placentula en las dos variedades, Fallacia pygmaea, Gomphonema parvulum, Nitzchia amphibia, Surirella ovalis y Ulnaria ulna. En general estas especies fueron citadas como cosmopolitas y representan organismos habituales de diversos sistemas acuáticos, como varios embalses de la región del NOA (Martínez De Marco et al., 2018; Seeligmann et Tracanna, 1994, entre otros). Cabe resaltar que las características diferenciales entre esta laguna y los embalses son varias y notorias (como tamaño, profundidad, etc), sin embargo, también presentan algunas similitudes por ser sistemas leníticos y alojar una biota equivalente.

Las asociaciones algales desarrolladas en tiempos y lugares comunes determinan el resultado de factores intrínsecos que afectan la composición y estado del ambiente acuático que pueden ser reversibles o no en relación a la salud biológica del ecosistema. En particular las taxocenosis de diatomeas son diversas y desde hace tiempo se conoce que su distribución está muy relacionada con la química del agua, la estacionalidad y la geología (Battarbee, 1986). La muerte celular con la consecuente caída de los frústulos silíceos ocasiona depósitos de sedimentos, principalmente en los sistemas leníticos. Los frústulos son resistentes al deterioro producido por acción bacteriana, erosión e incluso la presión mecánica de los mismos sedimentos depositados sobre ellos. De esta manera, los sedimentos ricos en diatomeas se transforman en un reservorio o archivo de la historia ambiental local, que puede ser utilizada para realizar comparaciones con las taxocenosis de diatomeas actuales.

En este trabajo se citan por primera vez para Tucumán a Nitschia inconspicua y Stauroneis anceps. Aunque la información es preliminar, la elevada riqueza diatomológica enfatiza la necesidad de intensificar los estudios taxonómicos, morfológicos, ecológicos y su empleo como bio-proxies de este grupo de microalgas. En el caso de las algas verdeazuladas y verdes, su frecuencia fue relativamente baja y las especies encontradas resultaron comunes a las mencionadas en sistemas como los embalses del Cadillal, La Angostura, Escaba y Termas de Río Hondo (Seeligmann et Tracanna, 1994, 2009; Martínez De Marco et al., 2018; Tracanna et Martínez De Marco, 1997). Consecuente a estos resultados es necesario realizar muestreos posteriores que permitirán acrecentar la riqueza de estos dos grupos de microalgas.

Debido a la falta de antecedentes limnológicos y ficológicos de esta laguna este trabajo contribuye a ampliar los conocimientos de la biodiversidad de microalgas del Noroeste de Argentina, lo que servirá como línea de base para posteriores investigaciones multidisciplinarias en este sistema lenítico de Yungas.

\section{Agradecimientos}

Agradecemos la colaboración en el campo de Juan Arce y Juan Ignacio Arce. El presente trabajo es una contribución al proyecto PIUNT G 635 de la Universidad Nacional de Tucumán.

\section{Bibliografía}

Anagnostidis, K. \& Komárek, J. (1988). Modern approach to the classification system of cyanophytes. 3- Oscillatoriales. Archiv für Hydrobiologie, Supplement 80, Algological Studies, 50-53: $327-$ 472 .

Battarbee, R. W. (1986). Diatom Analysis. En Berglund, B. E. (ed.), Handbook of Holocene Paleoecolgy and Paleohidrology, pp. 527-570. John Wiley \& Sons Ltd., Chichester.

Casado, S. \& Montes, C. (1995). Guía de los lagos y humedales de España. Ed. J. M. Reyero, Madrid.

Chapin, F. S., Zavaleta, E. S., Eviner, V. T., Rosamond, L. N., Vitousek, P. M. \& H. L. Reynolds. (2000). Consequences of changing biodiversity. Nature 405: 234-242. https://doi.org/10.1038/35012241 
Chen, I.-C., Shiu, H.-J., Benedick, S., Holloway, J. D., Chey, V. K., Barlow, H. S. \& Thomas, C. D. (2009). Elevation increases in moth assemblages over 42 years on a tropical mountain. Proceedings of the National Academy of Sciences of the United States of America 106: 1479-1483.

http://doi.org/10.1073/ pnas.0809320106

Desikachary, T. V. (1959). Cyanophyta. Indian Council of Agricultural Research, New Delhi.

Falkowski, P. G., Lows, E. A., Barber, R. T. \& Murray, J. W. (2003). Phytoplankton and their role in primary new, and export production. En Fasham, M. J. R. (ed.), Ocean Biogeochemistry: The role of the Ocean Carbon Cycle in global Change, pp. 99-121. Springer, Berlín. https://doi.org/10.1007/978-3-642-55844-3_5

Field, C. B., Behrenfeld, M. J., Randerson, J. T. \& FAlKowski, P. G. (1998). Primary producción of the Biosphere: Integrating terrestrial and Oceanic componentes. Science 281: 237-240. https://doi.org/10.1126/science.281.5374.237

Fernández, D. S. (2009). Eventos de remoción en masa en la Provincia de Tucumán: tipos, características y distribución. Revista de Asociación Geológica Argentina 65: 748-759.

Foden, W. B., Butchart, S. H. M., Stuart, S. N., Vié, J.-C., Aкçakaya, H. R., Angulo, A. \& Mace, G. M. (2013). Identifying the world's most climate change vulnerable species: a systematic trait-based assessment of all birds, amphibians and corals. PloS One 8: e65427. http://doi.org/10.1371/journal.pone.0065427

Frémy, P. (1930). Les Myxophycées de l'Afrique équatoriale française. Archives de Botanique, Mémoires 3: 1-508.

García Rodríguez, J., Molina Astudillo, F. I., Miranda, E., Soriano Salazar, M. B. \& Díaz Vargas, M. (2014). Variación fitoplanctónica en un lago urbano del municipio de Cuernavaca, Morelos México. Acta Universitaria 25: 3-11. https://doi.org/10.15174/au.2015.646

González Achem, A. L., Seeligmann, C. \& Alderete, M. (2014). Variaciones espacio-temporales de la flora diatomológica en laguna de los Pozuelos (Jujuy, Argentina). Boletín de la Sociedad Argentina de Botánica 49: 177-193.

https://doi.org/10.31055/1851.2372.v49.n2.7839

GRAU, R. (2010). Ecología de la interfase natural - urbana. San Javier - Tucumán como modelo de estudio. En Grau H. R. (ed.), Ecología de una interfase naturalurbana. La sierra de San Javier y el Gran San Miguel de Tucumán, pp. 11-18. Edunt, Tucumán.
Groombridge, B. (1992). Global Biodiversity: status of the earth's living resources : a report. Chapman and Hall, London.

Guarrera, S. A. \& Kühnemann O. (1949). Catálogo de las Chlorophyta y Cyanophyta de agua dulce de la República Argentina. Lilloa 19: 219-318.

Guiry, M. D. \& Guiry, G. M. (2020). AlgaeBase. Worldwide electronic publication, National University of Ireland, Galway. http://www.algaebase.org.

Hartley, B., Barber, H. G., Carter, J. R. and Sims, P. A. (1996). An atlas of British diatoms. Biopress Ltd, Bristol.

Huber-Pestalozzi, G. (1983). Das phytoplankton des Süßwassers. Systematik und Biologie. 7 Teil, 1 Häfte: Chlorophyceae (Grünalgen) Ordnung Chlorococcales. En KomÁreK J. \& F. Bohuslav (ed.) Die Binnengewässer Band XVI. E. Schweizerbart'sche Verlasbuchhandlung, Stuttgart, Germany.

Isasmendi, S. C., Seeligmann, C. T., Martínez De Marco, S. N. \& Tracanna, B. C. (2002). Flora diatomológica de un canal de descarga de desechos mineros (Tucumán - Argentina). Boletín de la Sociedad Argentina de Botánica 37: 41-49.

IsASMENDI, S. C. (2016). Estudio del fitoplancton y epiliton de ríos de Tafí del Valle (Tucumán, Argentina). Tesis Doctoral, Universidad Nacional de Tucumán, Argentina. 307 pp.

JoHn, D. M. (1994). Biodiversity and conservation: an algal perspective. The Phycologist 38: 3-15.

Komárek, J. \& Anagnostidis K. (1999). Cyanoprokaryota, 1: Chroococcales. En Ettl, H., G. Gärtner, H. Heynig \& D. Möllenhauer (eds.), Süßwasserflora von Mitteleuropa, 19. Gustav Fischer Verlag, Stuttgart.

Komárek, J. \& Anagnostidis K. (2005). Cyanoprokaryota. 2. Oscillatoriales. En Büdel B., Krienitz L., Gärtner G. \& M. Schagerl (eds.), Süsswasserflora von Mitteleuropa, 19/2. Elsevier/ Spektrum, Heidelberg.

Krammer, K. \& Lange-Bertalot, H. (1986). Bacillariophyceae. 1. Teil: Naviculaceae En ETtL, H., Gerloff J., H. Heynig \& D. Mollenhauer (eds.) Süsswasserflora von Mitteleuropa, Band 2/1. Gustav Fisher Verlag, Jena.

Krammer, K. \& Lange-Bertalot, H. (1988). Bacillariophyceae. 2. Teil: Bacillariaceae, Epithemiaceae, Surirellaceae En Etтl, H., J. Gerloff, H. Heynig \& D. Mollenhauer (eds.) Süsswasserflora von Mitteleuropa, Band 2/2. Gustav Fisher Verlag, Jena. 
Krammer, K. \& Lange-Bertalot, H. (1991). Bacillariophyceae. 3 Teil: Centrales, Fragilariaceae, Eunotiaceae. En Ettr, H., Gerloff J., H. Heynig \& D. Mollenhauer (eds.) Süsswasserflora von Mitteleuropa Band 2/3. Gustav Fischer Verlag, Stuttgart \& Jena.

Krammer, K. \& Lange-Bertalot, H. (2000). Bacillariophyceae. 3. Teil: Centrales, Fragilariaceae, Eunotiaceae. En Ettr, H., Gerloff, J., Heynig, H. \& Mollenhauer, D. (eds.). Süsswasserflora von Mitteleuropa 2. Gustav Fisher Verlag, Stuttgart, Germany.

Krammer, K. \& Lange-Bertalot, H. (2004). Bacillariophyceae. 4. Teil: Achnanthaceae. Kritische Ergänzungen zu Achnanthes s. 1., Navicula s. str., Gomphonema. Gesamtliteraturverzeichnis Teil 1-4. En Ettl, H.; Gärtner, G.; Gerloff, J.; Heynig, H. \& Mollenhauer, D. (eds.), Süßwasserflora von Mitteleuropa. Band 2/4. Spektrum Akademischer Verlag, Heidelberg.

Lange-Bertalot, H. (1999). Neue Kombinationen von Taxa aus Achnanthes Bory (sensu lato). Iconographia Diatomologica 6: 276-289.

Levkov Z. (2009) Amphora sensu lato. En LangeBertalot, H. (ed.) Diatoms of Europe 5: 5-916.

Lomáscolo, T., Grau, A. \& Brown, A. (2014). Áreas Protegidas de Tucumán. Ediciones del Subtrópico, Tucumán.

Luchini, L. \& Verona C. A. (1972). Catálogo de las diatomeas argentinas. I. Comisión de Investigaciones Científicas de la Provincia de Buenos Aires. Monografía 2, La Plata.

Luque, M. (1998). Comunidad fitoplanctónica y epilítica del río Piedra Blanca (Córdoba, Argentina). Tesis de Magister, Universidad Nacional del Litoral, Argentina. $117 \mathrm{pp}$.

Maidana, N. I. \& Herbst N. (1989a). Diatomeas (Bacillariophyceae) de la provincia de Santiago del Estero (Argentina) I. Darwiniana 29: 47-62.

Maidana, N. I. \& Herbst N. (1989b). Diatomeas (Bacilliariophyceae) de la laguna Nostra (Cumbres Calchaquíes), Provincia de Tucumán, Rep. Argentina. Darwiniana 29: 63-76.

Maidana, N. I., Vigna, M. S. \& Mascitti, V. (1998). Ficoflora de la Laguna Pozuelos (Jujuy, Argentina) I: Bacillariophyceae. Boletín de la Sociedad Argentina de Botánica 33: 171-179.

Maidana, N. I. \& Seeligmann, C. (2006). Diatomeas (Bacillariophyceae) de ambientes acuáticos de altura de la provincia de Catamarca, Argentina II. Boletín de la Sociedad Argentina de Botánica 41: 1-13.
Maidana, N. I., Seeligmann, C. \& Morales M. R. (2011). El género Navicula sensu stricto (Bacillariophyceae) en humedales de altura de Jujuy, Argentina. Boletín de la Sociedad Argentina de Botánica 46: 13-29.

Mancini, M., Crichigno, S., Ortiz, M. \& Haro, J. G. (2012). Lagos urbanos: Importancia, dinamismo y multiplicidad de usos. El caso del lago Villa Dalcar. Biología Acuática 27: 175-189.

ManN, D. G. (1999). The species concept in diatoms. Phycologia 38: 437-495. https://doi.org/10.2216/i0031-8884-38-6-437.1

Martínez de Fabricius, A. L. (1996). Bacillariophyceae del Río Cuarto, Provincia de Córdoba. Argentina. Tesis Doctoral, Universidad Nacional de La Plata, Argentina. $290 \mathrm{pp}$.

Martínez De Marco, S. N. (2009). Estudio de la comunidad fitoplanctónica en ríos de la cuenca superior del río Salí (Tucumán, Argentina). Tesis Doctoral FCN e IML, Universidad Nacional de Tucumán, Argentina. 226 pp.

Martínez De Marco, S. N., Tracanna B. C., Isasmendi, S. C., Alderete, M. del C., Taboada, M. de los Á. \& Mirande, V. (2018). Evaluación del fitoplancton del Embalse Escaba (Tucumán - Argentina) para caracterizar la calidad de sus aguas. Boletín de la Sociedad Argentina de Botánica 53: 521-42. https://doi.org/10.31055/1851.2372.v53.n4.21976

Metzeltin, D. \& Lange-Bertalot, H. (1998). Tropical diatoms of South America I: about 700 predominantly rarely known or new taxa representative of the neotropical flora. Iconographia Diatomologica 5: 1-695.

Metzeltin, D. \& Lange-Bertalot, H. (2007). Tropical diatoms of South America II: special remarks on biogeographic disjunction. Iconographia Diatomologica 18: 1-877.

Metzeltin, D., Lange-Bertalot, H. \& García-Rodríguez, F. (2005). Diatoms of Uruguay taxonomy-biogeographydiversity. Iconographia Diatomologica 15: 1-736.

Mirande, V. \& Tracanna, B. C. (1995). Estudio cualitativo del fitoplancton del embalse Río Hondo (Argentina): I. Cryptogamie Algologie 16: 211-232.

Mirande, V. (2006). Riqueza del fitoplancton en el arroyo Calimayo (Tucumán, Argentina). Lilloa 43: 61-86.

Mirande, V. \& Tracanna, B. C. (2003). El fitoplancton del río Gastona (Tucumán, Argentina) y su relación con la calidad del agua. Boletín de la Sociedad Argentina de Botánica 38: 51-64.

Mirande, V. \& Tracanna, B. (2015). Cianobacterias de humedales de altura del NOA. Lilloa 52: 122-141. http://www.lillo.org.ar/journals/index.php/lilloa/article/ view $/ 322$ 
Moraña, L. B. (1998). Estudio de la calidad del agua de un subsistema de ríos de la provincia de Salta sometida a acción antrópica. Tesis de Magister, Universidad Nacional del Litoral, Argentina. 87 pp.

Montoya Moreno, Y. \& Aguirre, N. (2013). Dinámica del Ensamblaje Algal epifítico en el sistema de planos inundables de Ayapel a través del pulso de inundación. Revista U.D.C.A Actualidad \& Divulgación Científica 16: 491-500. https://doi.org/10.31910/rudca.v16.n2.2013.923

Moyano, E. N. (2006). Paleontología y Estratigrafía de Sedimentitas Lacustres Cuaternarias, Quebrada de la Hoyadita, Valle de La Sala, Tucumán. Seminario de la Carrera Geología Universidad Nacional de Tucumán, Argentina. 53 pp.

Nieva, Á. M., Seeligmann, C. T. \& Maidana, N. I. (2019). Diatomeas perifíticas de un río de montaña (Tucumán, Argentina). Lilloa 56: 64-91. http://doi.org/10.30550/j.lil/2019.56.1/5

Nieto Peñalver, M. C., Ovando, X. M. C., Loyola, R., Izquierdo, A. E. \& Romero, F. (2017). The role of macroinvertebrates for conservation of freshwater systems. Ecology and Evolution 7: 5502-5513. https://doi.org/10.1002/ece3.3101

Olson, D. (2001). Terrestrial ecoregions of the world: A new map of life on Earth. BioScience 51: 933-938. https://doi.org/10.1641/00063568(2001)051[0933:TEOTWA]2.0.CO;2

PAtrick, R. (1961). Diatoms (Bacillariophyceae) from the alimentary tract of Phoenicoparrus jamesi (Sclater). Postilla 49: 43-55.

Patrick, R. M. \& Reimer, C. W. (1966). The Diatoms of the United States exclusive of Alaska and Hawaii. Volume 1. Fragilariaceae, Eunotoniaceae, Achnanthaceae, Naviculaceae. Monographs of the Academy of Natural Sciences of Philadelphia 13: 688 pp. https://doi.org/10.2307/1351135

Patrick, R. M. \& Reimer, C. W. (1975). The diatoms of the United States exclusive of Alaska and Hawaii. Volume 2. Part 2. Entomoneidaceae, Cymbellaceae, Gomphonemaceae, Epithemiaceae. Monographs of the Academy of Natural Sciences of Philadelphia 13: 213 pp.

Pedroche, F. F. (2000). Biodiversidad. ¡Divino tesoro!. En Kwiatkowska, T. \& R. L. Wilchis (eds.), Ingeniería genética y ambiental, pp. 153-168. México.

Prescott, G. W. (1961). Algae of Western Great Lakes Area. W. M. C. Brown Company Publishers, Dubuque.
Quiroga, P. \& Grau, A. (2020). Caracterización de la vegetación de la sierra de San Javier. En Julia, J. P., Quiroga, P., Barrionuevo O. A. (eds.), Historia Natural de las Yungas de Tafí Viejo, pp. 10-13. Tafí Viejo ediciones, Tafí Viejo. Libro digital, PDF.

Quirós, R. \& Drago, E. (1999). The environmental state of Argentinean lakes: An overview. Lakes \& Reservoirs. Research and Management 4: 55-64. https://doi.org/10.1046/j.1440-1770.1999.00076.x

Raxworthy, C. J., Pearson, R. G., Rabibisoa, N., Rakotondrazafy, A. M., Ramanamanjato, J. B., Raselimanana, A. P. \& Stone, D. A. (2008). Extinction vulnerability of tropical montane endemism from warming and upslope displacement: A preliminary appraisal for the highest massif in Madagascar. Global Change Biology 14: 1703-1720. http://doi.org/10.1111/ j.1365-2486.2008.01596.x

Reichardt, E. (1999). Zur Revision der Gattung Gomphonema. Die Arten um G. affine/insigne, G. angustatum/micropus, G. acuminatum y sowie gomphonemoide Diatomeen aus dem Oberoligozán in Böhmena. En H. LANGeBertalot. (ed.), Iconographia Diatomologica. Annotated Diatom Micrographs Vol. 8 Taxonomy, pp. 203. A. R. G. Gantner, Ruggell, Liechtenstein. Reynolds, C. S. (2006). The Ecology of Phytoplankton. Cambridge University Press, Cambridge.

Round, F. E., Crawford, R. M. \& Mann, D. G. (1990). The diatoms. Biology and morphology of the genera. Cambridge University Press, Cambridge, England.

Rumrich, U., Lange-Bertalot, H. \& Rumrich, M. (2000). Diatomeen der Anden von Venezuela bis Patagonien/Tierra del Fuego. En H. LangeBertalot (ed.), Iconographia Diatomologica 9, pp.1-672. A. R. G. Gantner Verlag K. G., Ruggell.

Sala, O. E., Chapin, F. S. \& Armesto, J. J. (2000) Global Biodiversity Scenarios for the Year 2100. Science 287: 1770-1774. https://doi.org/10.1126/science.287.5459.1770

SAlusso, M. M. (1998). Evaluación de la calidad del agua de dos ríos del valle de Lerma (Salta) sometidos a acción antrópica. Tesis de Magister, Universidad Nacional del Litoral, Argentina. 84 pp.

SAlusso, M. M. (2005). Evaluación de la calidad de los recursos hídricos superficiales en la Alta Cuenca del Juramento (Salta). Tesis Doctoral, Universidad Nacional de Buenos Aires, Argentina. 209 pp. 
Schröter, D., Cramer, W., Leemans, R., Prentice, I. C., Araújo, M. B. \& Arnell, N. W. (2005). Ecosystem service supply and vulnerability to global change in Europe. Science 310: 1333-1337. https://doi.org/10.1126/science.1115233

Seeligmann, C. (1998). Evaluación de la estructura y dinámica ficológica del río Salí (TucumánArgentina), en relación al impacto de la contaminación antropogénica. Tesis Doctoral, Universidad Nacional de Tucumán, Argentina. 199 pp. (Inédita).

Seeligmann, C. \& Maidana, N. (2003). Diatomeas (Bacillariophyceae) de ambientes acuáticos de altura de la provincia de Catamarca (Argentina). Boletín de la Sociedad Argentina de Botánica 38: 39-50.

Seeligmann, C. \& Maidana, N. I. (2013). ¿Existen especies de Navicula (Bacillariophyta) exclusivas de ambientes de alta montaña en Argentina? Boletín de la Sociedad Argentina de Botánica 38: 39-50.

Seeligmann, C., Maidana, N. I. \& Morales, M. (2008). Diatomeas (Bacillariophyceae) de humedales de altura de la provincia de Jujuy, Argentina. Boletín de la Sociedad Argentina de Botánica 43: 1-17.

Seeligmann, C. T. \& Tracanna, B. C. (1994). Limnología del embalse El Cadillal (Tucumán-Argentina): II: Estudio cualitativo del fitoplancton. Cryptogamie Algologie 15: 19-35.

Seeligmann, C. T. \& Tracanna, B. C. (2009). Dinámica del fitoplancton en un embalse de alta cota del Noroeste Argentino (Tucumán). Limnetica 28: 105 124.

Seeligmann, C., Tracanna, B. C., Martínez De Marco, S. \& IsASMEnd, S. (2001). Algas fitoplanctónicas en la evaluación de la calidad de agua de sistemas lóticos en el Noroeste Argentino. Limnetica 20: 123-133.

Silva, P. C. (1992). Geographic patterns of diversity in benthic marine algae. Pacific Science 46: 429-437.

StARmaCH, K. (1966). Cyanophyta - Sinice. Glaucophyta - Glaukofity. En Starmach, K. (ed.), Flora Słodkowodna Polski. 2. PWN, Warszaw-Kraków.

TABOADA, M. DE Los Á. (2017). Estudio de la Ficoflora como Bioindicadora del Estado Ecológico en Sistemas lóticos de Tucumán. Evaluación del
Impacto Antrópico. Tesis Doctoral, FCN e IML, Universidad Nacional de Tucumán, Argentina. 272 pp.

Taboada, M. de los Á., Gultemirian, M. De L., Martínez De Marco, S. N. \& Tracanna, B. C. (2015). Ficoflora Epilítica y variables ambientales del Arroyo Calimayo (Tucumán-Argentina). Boletín de la Sociedad Argentina de Botánica 50: 467-480. https://doi.org/10.31055/1851.2372.v50.n4.12910

Taboada, M. de los Á., Martínez De Marco, S. N. \& Tracanna, B. C. (2016). Biodiversidad Epilítica de un arroyo subtropical del Noroeste Argentino. Lilloa 53: $10-22$.

Tell, G. (1985). Catálogo de las algas de agua dulce de la República Argentina. Bibliotheca Phycologica, Band 70. Ed. J. Cramer, Vaduz.

Tracanna, B. C. (1982). Estudio taxonómico de las Chlorophyta de Tucumán (incluidas algunas consideraciones ecológicas). Opera Lilloana 32.

Tracanna, B. C. (1985). Algas del Noroeste Argentino (excluyendo a las Diatomophyceae). Opera Lilloana 35: 1-136.

Tracanna, B. C. \& Martínez De Marco, S. (1997). Ficoflora del río Salí sus tributarios en áreas del embalse Dr. Gelsi (Tucumán-Argentina). Natura Neotropicalis 28: 23-38. https://doi.org/10.14409/natura.v1i28.3694

Viollaz, C., Martínez, S., Lomáscolo, T. \& Pacheco, S. (2015). Lineamientos de base para una planificación de desarrollo sustentable y sostenible del valle del Siambón. Revista científica del IDITec 4: 33-50.

Villafañe, V. \& ReID, F. (1995). Métodos de Microscopía para la cuantificación del fitoplancton. En AlveAL, K., M. Ferrario, E. Oliveira \& E. Sar (eds.), Manual de métodos Ficológicos, pp.169-185. Universidad de Concepción, Chile.

Vouilloud, A. (2003). Catálogo de diatomeas continentales y marinas de Argentina. Versión 1.0. En soporte magnético. Asociación Argentina de Ficología, La Plata.

Wetzel, R. G. (1981). Limnología. Omega, Barcelona.

Wolf, H. D. (1982). Methods of coding of ecological data from Diatom for computer utilization. Mededelingen Rijks Geologische Dienst 36: 95-110. 